\title{
WISEP J004701.06+680352.1: AN INTERMEDIATE SURFACE GRAVITY, DUSTY BROWN DWARF IN THE AB DOR MOVING GROUP
}

\author{
John E. Gizis ${ }^{1,9}$, Katelyn N. Allers ${ }^{2,10}$, Michael C. Liu ${ }^{3,10}$, Hugh C. Harris ${ }^{4}$, \\ JACQUEline K. FAherty ${ }^{5,6}$, AdAm J. Burgasser ${ }^{7}$, AND J. DAVy KirKPATRICK ${ }^{8}$ \\ ${ }^{1}$ Department of Physics and Astronomy, University of Delaware, Newark, DE 19716, USA \\ ${ }^{2}$ Department of Physics and Astronomy, Bucknell University, Lewisburg, PA 17837, USA \\ ${ }^{3}$ Institute for Astronomy, University of Hawaii, 2680 Woodlawn Drive, Honolulu HI 96822, USA \\ ${ }^{4}$ US Naval Observatory, Flagstaff Station, 10391 West Naval Observatory Road, Flagstaff, AZ 86001, USA \\ ${ }^{5}$ Department of Terrestrial Magnetism, Carnegie Institution of Washington 5241 Broad Branch Road NW, Washington, DC 20015, USA \\ ${ }^{6}$ Department of Astrophysics, American Museum of Natural History, Central Park West at 79th Street, New York, NY 10034, USA \\ ${ }^{7}$ Center for Astrophysics and Space Science, University of California San Diego, La Jolla, CA 92093, USA \\ ${ }^{8}$ Infrared Processing and Analysis Center, MS 100-22, California Institute of Technology, Pasadena, CA 91125, USA \\ Received 2014 March 9; accepted 2014 December 4; published 2015 January 30
}

\begin{abstract}
We present spectroscopy, astrometry, and photometry of the brown dwarf WISEP J004701.06+680352.1 (W0047+68), an unusually red field L dwarf at a distance of $12.2 \pm 0.4 \mathrm{pc}$. The three-dimensional space motion identifies it as a member of the AB Dor Moving Group, an identification supported by our classification of W0047+68 as intermediate surface gravity (INT-G) using the Allers \& Liu near-infrared classification system. This moving group membership implies near-solar metallicity, age $\sim 100-125 \mathrm{Myr}, M \approx 0.018 M_{\odot}$, and $\log g \approx 4.5$; the thick condensate clouds needed to explain the infrared spectrum are, therefore, a result of surface gravity that is lower than that of ordinary field brown dwarfs. From the observed luminosity and evolutionary model radius, we find $T_{\text {eff }} \approx 1300 \mathrm{~K}$, a temperature normally associated with early $\mathrm{T}$ dwarfs. Thick clouds are also used to explain the spectral properties of directly imaged giant planets, and we discuss the successes and challenges for such substellar models in matching the observed optical and infrared spectra. W0047+68 shows that cloud thickness is more sensitive to intermediate surface gravity than in most models. We also present a trigonometric parallax of the dusty L6 dwarf 2MASS J21481628+4003593. It lies at $8.060 \pm 0.036$ parsecs; its astrometry is consistent with the view that it is older and metal-rich.
\end{abstract}

Key words: brown dwarfs - infrared: stars - stars: individual (WISEP J004701.06+680352.1, 2MASS J21481628+4003593)

\section{INTRODUCTION}

One of the key challenges in substellar astronomy is to use observations of colors, absolute magnitudes, and spectra to deduce the fundamental physical parameters of brown dwarfs and gas giant planets. Determining the luminosity, effective temperature, surface gravity, composition, mass, and radius would allow many astrophysical applications, including testing formation scenarios for "planetary mass objects," but is difficult in part due to the importance of condensate cloud formation at substellar atmospheric temperatures. The vast majority of the nearly two thousand known field brown dwarfs can be described by a well-defined, one-dimensional spectral sequence of late-M, - $\mathrm{L},-\mathrm{T}$, and $-\mathrm{Y}$ dwarfs, with a dramatic change in cloud properties at the $\mathrm{L} / \mathrm{T}$ transition around $1400 \mathrm{~K}$ (see the review of Kirkpatrick 2005). It is now clear, however, that many of the directly imaged planetary mass companions have significant differences from ordinary field brown dwarfs. The companions 2M1207b (Chauvin et al. 2004; Patience et al. 2010) and HR 8799b, c, d, and e (Marois et al. 2008, 2010), are considerably redder than field brown dwarfs of similar

\footnotetext{
9 Visiting Astronomer, Kitt Peak National Observatory, National Optical Astronomy Observatory, which is operated by the Association of Universities for Research in Astronomy (AURA) under cooperative agreement with the National Science Foundation.

${ }^{10}$ Visiting Astronomer at the Infrared Telescope Facility, which is operated by the University of Hawaii under Cooperative Agreement no. NNX-08AE38A with the National Aeronautics and Space Administration, Science Mission Directorate, Planetary Astronomy Program.
}

temperature or luminosity. The planet $\beta$ Pic $\mathrm{b}$ has apparently normal near-infrared colors for a mid-L dwarf but very red midinfrared colors (Currie et al. 2013), and the $L^{\prime}$ magnitudes of the HR 8799 planets are also brighter than expected (Skemer et al. 2012). Spectra of the HR 8799 planets show considerable diversity, which may require non-equilibrium mixing, different cloud properties, and perhaps enhanced metals or non-solar abundances (Bowler et al. 2010; Barman et al. 2011; Marley et al. 2012; Konopacky et al. 2013; Oppenheimer et al. 2013), but distinguishing between these possibilities is difficult.

Rarer classes of field brown dwarfs, especially the unusually dusty ones, offer a way forward, as they can be more accessible to observations than planets. A number of systematic studies of young $(<150 \mathrm{Myr})$ late-M and early-L-type brown dwarfs (Kirkpatrick et al. 2008; Cruz et al. 2009; Patience et al. 2012; Faherty et al. 2013a, 2013b; Allers \& Liu 2013a; Manjavacas et al. 2014) have demonstrated their red colors and other spectral peculiarities, which can be ascribed to low-surfacegravity dusty atmospheres. Unfortunately, even cooler brown dwarfs are necessarily fainter and more difficult to discover. Gizis et al. (2012, hereafter Paper I) noted that there were then only five known field $\mathrm{L}$ dwarfs with $J-K>2.3$, and none had trigonometric parallaxes. The field is developing rapidly with new sky surveys, and the two currently reddest $(J-K=2.8)$ field L dwarfs known are PSO J318.5338-22.8603 (Liu et al. 2013b) and ULAS J222711-004547 (Marocco et al. 2014). ${ }^{11}$

\footnotetext{
11 All names are abbreviated after first use: PSO J318-22 for PSO J318.5338-22.8603, W0047+68 for WISEP J004701.06+680352, etc.
} 


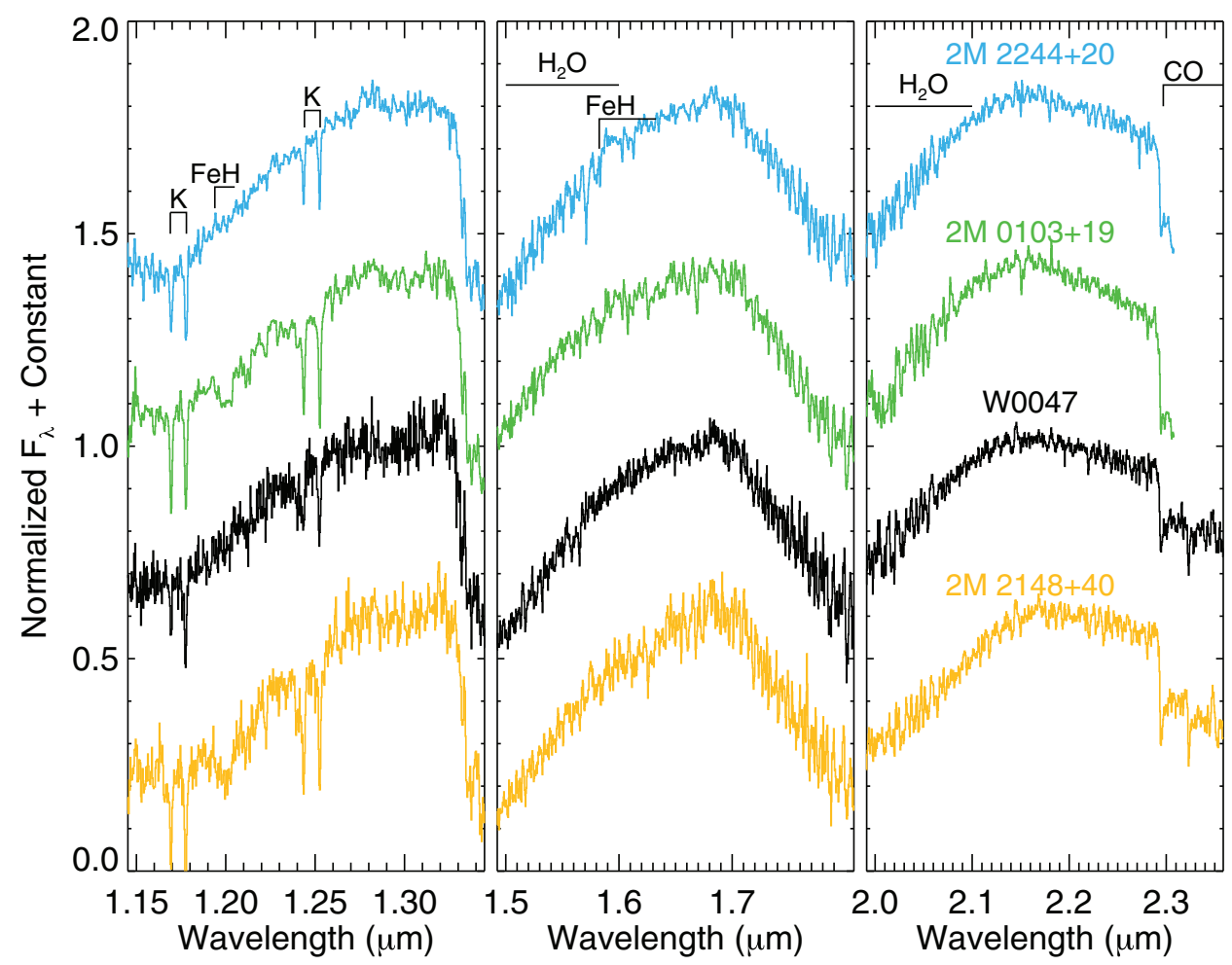

Figure 1. Moderate-resolution near-infrared IRTF spectrum of W0047+68 compared to other unusually red L dwarfs from Allers \& Liu (2013a).

PSO J318-22's trigonometric parallax, tangential velocity and unusual spectrum suggests it is a planetary-mass member of the $\beta$ Pic Moving Group (Zuckerman et al. 2001), and in many ways is similar to $2 \mathrm{M} 1207 \mathrm{~b}$. It has the faintest $J$-band absolute magnitude (14.8) of any L dwarf other than 2M1207b (16.13, Gizis et al. 2007). Besides PSO J318-22, the best studied of the extremely red L dwarfs are 2MASS J21481628+4003593 (Looper et al. 2008), which is thought to be old ( $\gtrsim 200 \mathrm{Myr}$ ), 2MASSW J2244316+204343 (Dahn et al. 2002), which has been proposed to be young (Kirkpatrick et al. 2008), and 2MASS J035523.37+113343.7, a dusty, red L-type dwarf with low surface gravity (Cruz et al. 2009; Faherty et al. 2013b; Liu et al. 2013a).

WISEP J004701.06+680352 (Paper I) was the reddest $(J-$ $\left.K_{s}=2.55 \pm 0.08\right)$ known $\mathrm{L}$ dwarf at the time of discovery. Although its low-resolution near-infrared spectrum resembles older L dwarfs (see Paper I), Thompson et al. (2013) have suggested that it is young and has low surface gravity on the basis of its moderate-resolution spectrum, and Gagné et al. (2014) argue that it and $2 \mathrm{M} 2244+20$ are probable members of the AB Dor Moving Group (ABDMG). In this paper, we present new observations and analysis of the brown dwarf W0047+68, including optical and near-infrared spectra, photometry, and a preliminary trigonometric parallax, as well as a parallax for $2 \mathrm{M} 2148+40$. We argue that W0047+68 is a bona fide member of the AB Dor Moving Group (ABDMG), and confront theoretical dusty models with our new observational constraints. We find that $2 \mathrm{M} 2148+40$ 's space motion is consistent with the view that it is older and metal-rich.

\section{DATA AND OBSERVATIONS}

\subsection{Near-infrared Spectra}

A moderate-resolution near-infrared spectrum covering the range of 0.8 to $2.4 \mu \mathrm{m}$ was obtained using the IRTF SpeX spectrograph (Rayner et al. 2003) in cross-dispersed mode (hereinafter SXD) on UT Date 2012 September 26. The resolution is 1200. The data were reduced using the facility pipeline Spextool (Cushing et al. 2004). Telluric features were corrected using a nearby A star using the method of Vacca et al. (2003). Spectra with the same observing setup were used by Allers \& Liu (2013a) to develop a spectroscopic classification system that is sensitive to surface gravity: the key indicators include the $\mathrm{K}_{\mathrm{I}}$ lines in the $J$ band and the $\mathrm{FeH}$ bands in $J$ and $H$ bands. In Figure 1, we compare our spectrum to other unusually red L dwarfs 2M2244+20, 2MASS J01033203+1935361 (Kirkpatrick et al. 2000; Faherty et al. 2012), and 2M2148+40. W0047+68 is similar to $2 \mathrm{M} 2244+20$, but has considerably lower surface gravity than 2M2148+40. Applying the Allers \& Liu (2013a) system, we classify W0047+68 as L7 INT-G (Table 1).

High-resolution near-infrared spectra of W0047+68 were obtained with Keck/NIRSPEC (McLean et al. 1998, 2000) on UT Date 2013 September 17 to determine W0047+68's radial velocity and projected rotational velocity $(v \sin i)$. Data were obtained using the N7 filter and $0.432 \times 12^{\prime \prime}$ slit $(3$ pixel dispersion), providing 1.99-2.39 $\mu \mathrm{m}$ spectroscopy in seven orders at a measured resolution of 25,000 $\left(\Delta v=12 \mathrm{~km} \mathrm{~s}^{-1}\right)$. Two dithered exposures of $1200 \mathrm{~s}$ were obtained at an airmass of 1.50, followed by observations of a nearby A star. Data in the order of 33, spanning 2.293-2.319 $\mu \mathrm{m}$, were extracted and analyzed due to the strong $\mathrm{CO}$ bands present in this band. We used a combination of REDSPEC ${ }^{12}$ and custom IDL routines that perform a forward-modeling Monte Carlo Markov Chain analysis of the extracted spectrum using solarmetallicity BT-Settl models (Allard et al. 2011) and a telluric absorption spectrum from Livingston \& Wallace (1991); this analysis will be described in detail in a forthcoming paper (A. J. Burgasser et al., in preparation; see Blake et al. 2010 for a similar algorithm). Figure 2 displays our extracted spectrum

\footnotetext{
12 See http://www2.keck.hawaii.edu/inst/nirspec/redspec/index.html.
} 


\section{Wavelength $(\mu \mathrm{m})$}
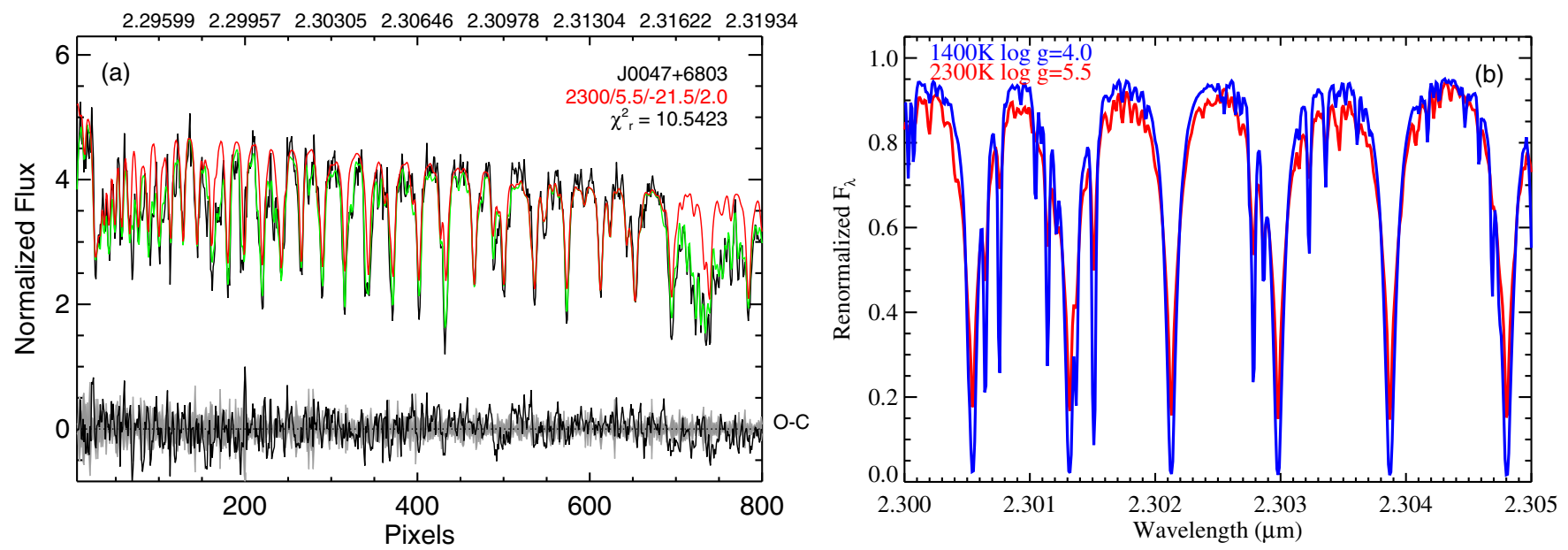

Figure 2. Analysis of our Keck/NIRSPEC spectrum of W0047+68 (black line). Left: the best-fit model (red line; $T_{\text {eff }}=2300 \mathrm{~K}$ and $\log g=5.5 \mathrm{cgs}$ ) has been broadened by $v \sin i=2 \mathrm{~km} \mathrm{~s}^{-1}$ and shifted by a heliocentric radial velocity of $-21.5 \mathrm{~km} \mathrm{~s}^{-1}$. The green line shows the combination of model and telluric absorption (from Livingston \& Wallace 1991) which best fits the data. Residuals are shown at bottom (black line) and are comparable to the noise ( $\pm 1 \sigma$ indicated by the gray region around zero flux). Right: a comparison of two BT-Settl (Allard et al. 2011) models, $T_{\text {eff }}=2300 \mathrm{~K}$ with $\log g=5.5$ and $T_{\text {eff }}=1400 \mathrm{~K}$ with $\log g=4.0$, illustrating that high-resolution spectra in this wavelength region can be used for velocity information but do not reliably constrain temperature.

Table 1

WISEP J004701.06+680352.1

\begin{tabular}{|c|c|c|}
\hline Parameter & W0047+6803 & Remarks \\
\hline Sp. type & L7 INT-G & Infrared, Allers \& Liu (2013a) system \\
\hline Sp. type & L7 pec & Optical, Kirkpatrick et al. (1999) system \\
\hline$\pi_{\mathrm{abs}}$ & $82 \pm 3$ & mas \\
\hline$\mu_{\text {rel }}$ & $434 \pm 3$ & $\operatorname{mas} \mathrm{yr}^{-1}$ \\
\hline$\theta$ & $117.0 \pm 0.5$ & degrees, east of north \\
\hline$v_{\text {rad }}$ & $-20.0 \pm 1.4$ & $\mathrm{~km} \mathrm{~s}^{-1}$ \\
\hline$v \sin i$ & $4.3 \pm 2.2$ & $\mathrm{~km} \mathrm{~s}^{-1}$ \\
\hline$z$ & $18.95 \pm 0.03$ & AB mag, Pan-STARRS \\
\hline$y$ & $18.12 \pm 0.03$ & AB mag, Pan-STARRS \\
\hline$U$ & $-8.6 \pm 1.0$ & $\mathrm{~km} \mathrm{~s}^{-1}$ \\
\hline$V$ & $-27.8 \pm 1.3$ & $\mathrm{~km} \mathrm{~s}^{-1}$ \\
\hline$W$ & $-13.6 \pm 0.5$ & $\mathrm{~km} \mathrm{~s}^{-1}$ \\
\hline$M_{J}$ & $15.17 \pm 0.11$ & 2MASS \\
\hline$M_{H}$ & $13.54 \pm 0.09$ & 2MASS \\
\hline$M_{K s}$ & $12.62 \pm 0.09$ & 2MASS \\
\hline$M_{W 1}$ & $11.47 \pm 0.08$ & WISE \\
\hline$M_{W 2}$ & $10.82 \pm 0.08$ & WISE \\
\hline$M_{W 3}$ & $9.71 \pm 0.10$ & WISE \\
\hline$m_{\text {bol }}$ & $16.30 \pm 0.03$ & Section 2.5 \\
\hline$L / L_{\odot}$ & $(3.58 \pm 0.29) \times 10^{-5}$ & Section 2.5 \\
\hline$B C_{J}$ & $0.70 \pm 0.08$ & \\
\hline$B C_{K}$ & $3.25 \pm 0.04$ & \\
\hline$M_{J}(\mathrm{MKO})$ & $15.05 \pm 0.11$ & Color term from spectrum, see Paper I \\
\hline$M_{H}(\mathrm{MKO})$ & $13.65 \pm 0.09$ & See Paper I \\
\hline$M_{K}(\mathrm{MKO})$ & $12.56 \pm 0.09$ & See Paper I \\
\hline$M_{M^{\prime}}$ & 10.6 & Using 2M2244, see Section 2.4 \\
\hline \multicolumn{3}{|c|}{ If $\mathrm{AB}$ Dor Association member } \\
\hline$T_{\text {eff }}$ & $\sim 1270$ & K, see Section 3.3 \\
\hline Age & $\sim 120$ & Myr \\
\hline Mass & $\sim 0.018$ & $M_{\odot}$ \\
\hline $\log g$ & $\sim 4.5$ & \\
\hline \multicolumn{3}{|c|}{ If older Galactic Disk member } \\
\hline$T_{\text {eff }}$ & $\sim 1410$ & $\mathrm{~K}$; see Section 3.3 \\
\hline Age & $\leqslant 1000$ & Myr \\
\hline Mass & $<0.055$ & $M_{\odot}$ \\
\hline $\log g$ & $\sim 5.0$ & \\
\hline
\end{tabular}




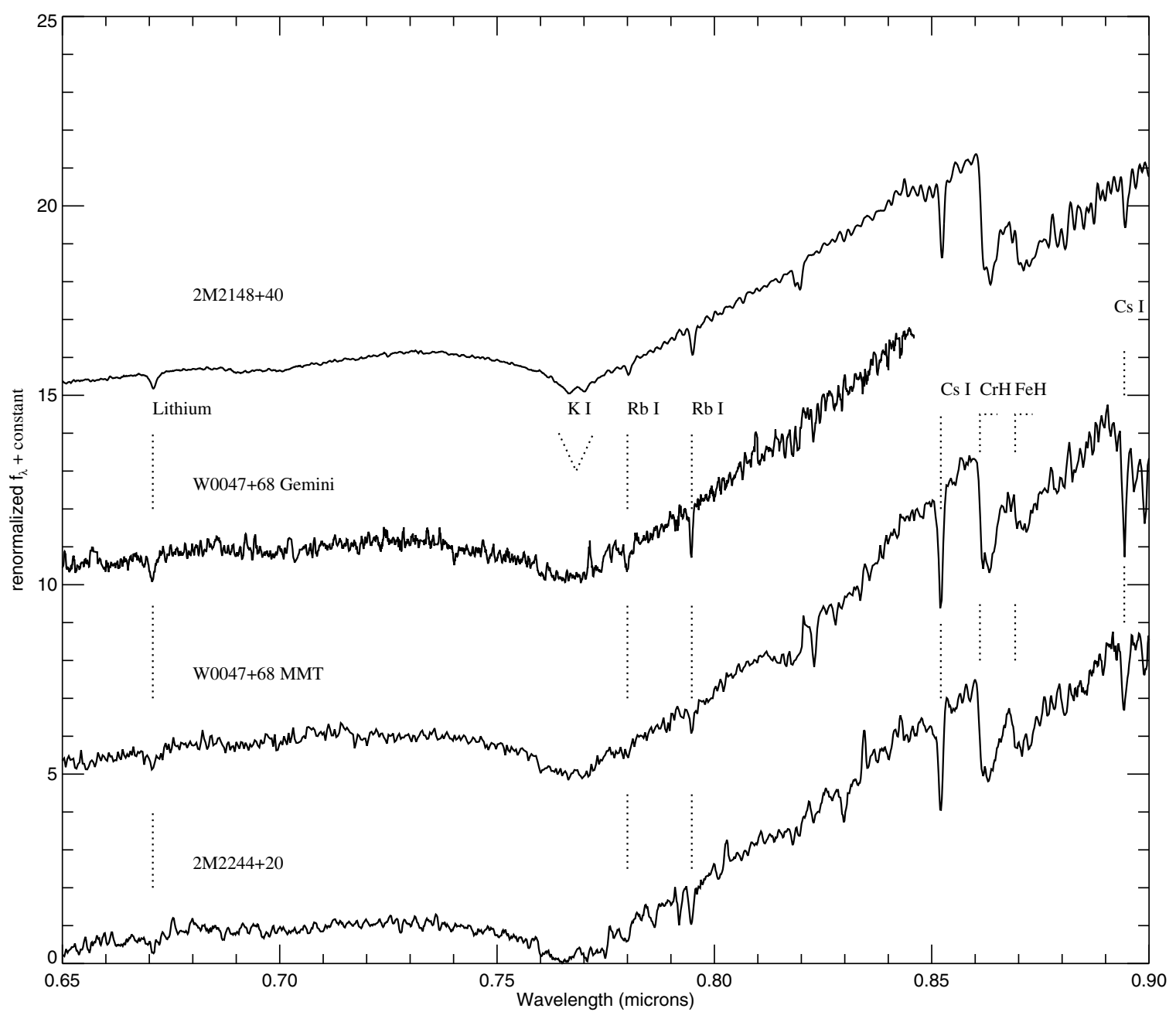

Figure 3. Optical spectra of W0047+68, along with the red L6.5 (opt) 2M2244+20 from Kirkpatrick et al. (2008) and the red L6 (opt) $2 \mathrm{M} 2148+40$ from Looper et al. (2008). All four spectra have been smoothed.

and the best-fit model. The individual best-fit model has $v_{\text {rad }}=$ $-21.5 \mathrm{~km} \mathrm{~s}^{-1}$ and $v \sin i=2 \mathrm{~km} \mathrm{~s}^{-1}$, but marginalizing over the fit parameters, including other temperatures and gravities, we find $v \sin i=4.3 \pm 2.2 \mathrm{~km} \mathrm{~s}^{-1}$ and a heliocentric radial velocity of $-20.0 \pm 1.4 \mathrm{~km} \mathrm{~s}^{-1}$, which we adopt as our best estimates for W0047+68. Our modeling requires higher resolution than is publicly available for the full BT-Settl grid; the subset of the grid that we use has $T_{\text {eff }}>2000 \mathrm{~K}$, hotter than we believe W0047+68 can possibly be. However, these CO lines are not very sensitive to temperature. We show in Figure 2(b) a comparison of the $T_{\text {eff }}=1400 \mathrm{~K}, \log g=4.0$ model (which we discuss later) to the hotter models used by the modeling routine at the highest resolution available. The lines are very similar, suggesting that the radial velocity and $v \sin i$, but not the temperature or gravity, can be extracted from the Keck data.

\subsection{Optical Spectra}

Optical (far-red) long-slit spectra were obtained with the MMT and Gemini-North telescopes. The MMT observations were on UT Date 2012 August 26 with the Red Channel spectrograph with grating 270, using three $600 \mathrm{~s}$ exposures. Conditions were non-photometric. This time was allocated by NOAO as program 2012B-0233. The wavelength coverage was 6170-9810 $\AA$ with a resolution of $\sim 12 \AA$, but we make no use of the spectrum redder than $9250 \AA$ due to strong telluric water absorption. The Gemini-North observations (Gemini program GN-2012B-Q-105) were on UT Date 2012 September 11 with the GMOS spectrograph (Hook et al. 2004) using grating R831. Four $600 \mathrm{~s}$ exposures were taken during cloudy conditions. The wavelength coverage was $6340-8460 \AA$ with a resolution of $\sim 2 \AA$, but for all plots, we smooth to a resolution of $\sim 10 \AA$ due to low signal-to-noise. The spectra were processed using standard IRAF tasks.

The MMT and Gemini spectra are shown in Figure 3, along with $2 \mathrm{M} 2244+20$, classified as L6.5 in the optical (Kirkpatrick et al. 2008), and $2 \mathrm{M} 2148+40$, classified as L7 in the optical (Looper et al. 2008). The core of the $\mathrm{KI}$ doublet and the $\mathrm{CrH}$ and $\mathrm{FeH}$ features are shown in Figure 4 along with the original Kirkpatrick et al. (1999) L6-L8 standard star spectra. The CrH and $\mathrm{FeH}$ features most strongly support an L7 classification, and the $\mathrm{K}_{\mathrm{I}}$ doublet and overall appearance of the spectrum is consistent with this. It is evident, however, that the Rb I and Cs I lines are much weaker in W0047+68 than in the standard, or typical, L6-L8 dwarfs (Kirkpatrick et al. 1999, 2000), suggesting that a classification of "L7 peculiar" is appropriate. In Figure 5, we show the same comparison to the unusually red L dwarfs 2M0355+11 (Cruz et al. 2009), 2M2148+40 (Looper et al. 2008), and 2M2244+20 (Kirkpatrick et al. 2008). These spectra were classified as "L5 $\gamma$," L6, and L6.5 respectively. 


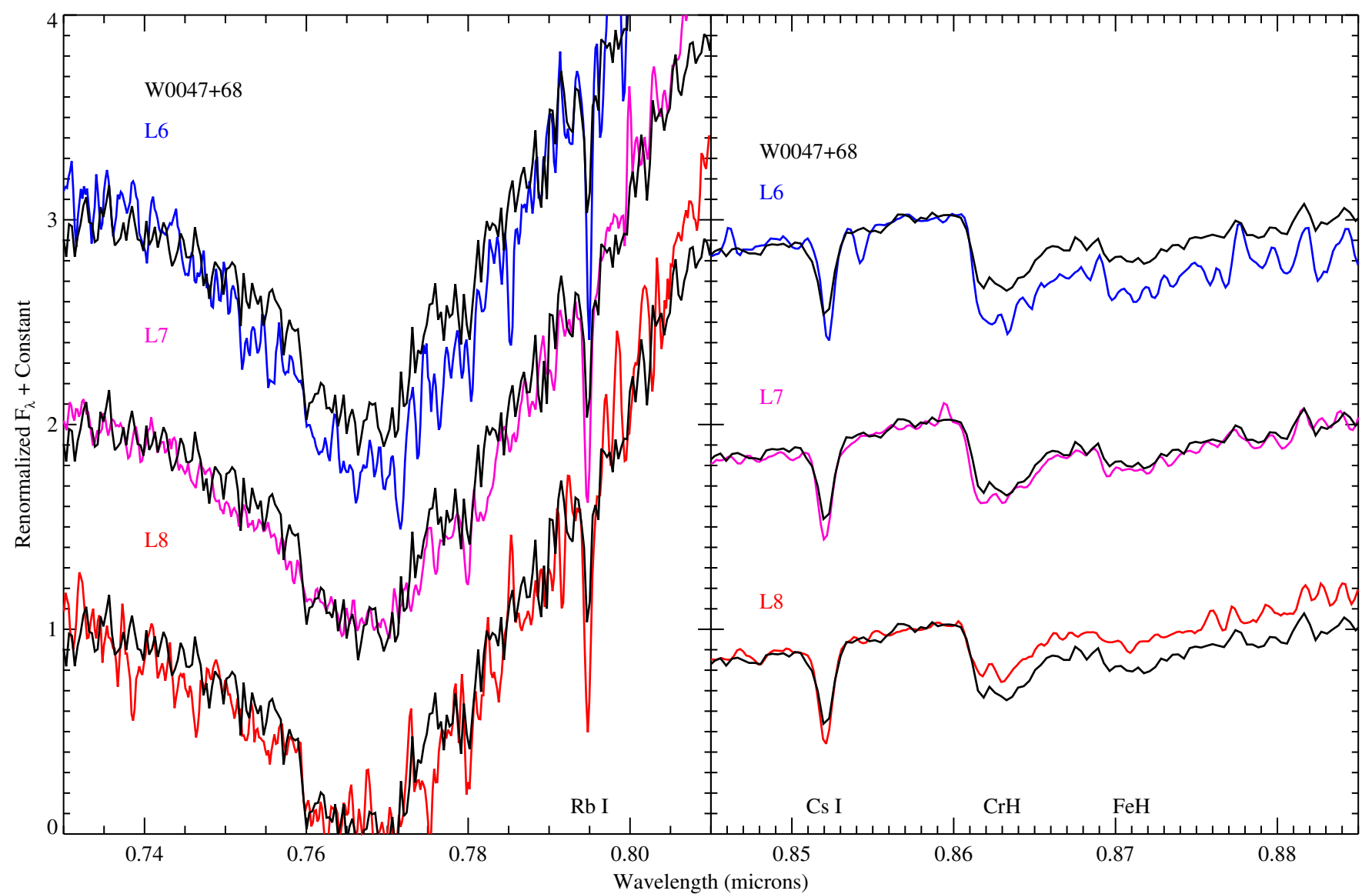

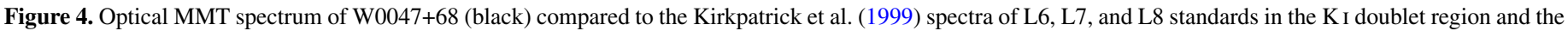

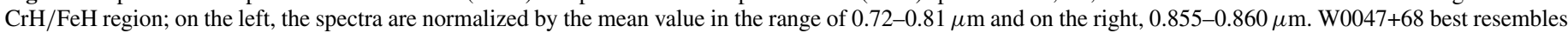

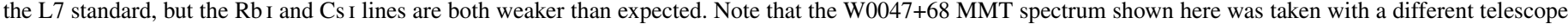
and instrument, but we have smoothed the data to match resolutions.

The weak Cs I is also evident in 2M2148+40 and 2M2244+20. W0047+68's alkali lines are stronger than in the low gravity $2 \mathrm{M} 0355+11$, but $2 \mathrm{M} 0355+11$ is a warmer type. Given our nearinfrared classification of INT-G, the atomic line peculiarities hint that W0047+68 might be classified as $\mathrm{L} 7 \beta$ in the optical, but we believe that extending the Cruz et al. (2009) system to such a late-type object would be premature without more objects. Both W0047+68 observations show lithium absorption, but no detectable $\mathrm{H} \alpha$ emission.

\subsection{Astrometry}

W0047+68 is being observed as part of the USNO optical parallax program, which is described in Dahn et al. (2002). Based on 23 frames taken in two seasons, the preliminary absolute parallax is $82 \pm 3$ mas with relative proper motion $\mu=434 \pm 3{\text { mas } y r^{-1}}^{-1}$ at position angle $\theta=117.0 \pm 0.5$. We derive the $U, V, W$ space velocity of W0047+68 using the observed radial velocity and astrometry and the IDL code gal_uvw.pro, except that we adopt the traditional astronomer convention that $U$ is positive toward the Galactic center. Note that $W$ is strongly constrained $\left( \pm 0.5 \mathrm{~km} \mathrm{~s}^{-1}\right)$ by the astrometry because of the position on the sky $(l=122.5, b=+05.2)$. We note that the absolute magnitude at the $H$ band agrees with that of typical L7 dwarfs (Dupuy \& Liu 2012); W0047+68 is fainter ("sub-luminous") at shorter wavelengths ( $J$ band) and brighter ("over-luminous") at longer wavelengths ( $K$, WISE).
Table 2

2MASS J21481628+4003593

\begin{tabular}{lcl}
\hline \hline Parameter & 2M2148+40 & \multicolumn{1}{c}{ Remarks } \\
\hline Sp. Type & L6 FLD-G & Infrared, Allers \& Liu (2013a) \\
Sp. Type & L6 & Optical, Looper et al. (2008), \\
$\pi_{\text {abs }}$ & $124.07 \pm 0.55$ & mas \\
$\mu_{\text {rel }}$ & $901.9 \pm 0.3$ & mas \\
$U$ & $-34.0 \pm 0.5$ & $\mathrm{~km} \mathrm{~s}^{-1}$ \\
$m_{\text {bol }}$ & $15.12 \pm 0.03$ & Section 3.3 \\
$L / L_{\odot}$ & $(4.64 \pm 0.14) \times 10^{-5}$ & Section 2.5 \\
$M_{J}$ & $14.62 \pm 0.03$ & 2MASS \\
$M_{H}$ & $13.25 \pm 0.03$ & $2 \mathrm{MASS}$ \\
$M_{K s}$ & $12.23 \pm 0.03$ & $2 \mathrm{MASS}$ \\
$M_{W 1}$ & $11.21 \pm 0.02$ & WISE \\
$M_{W 2}$ & $10.70 \pm 0.02$ & WISE \\
$M_{W 3}$ & $10.13 \pm 0.06$ & WISE \\
\hline
\end{tabular}

Few trigonometric parallaxes of unusually red L5 or later dwarfs exist, complicating comparisons of W0047+68 to other objects. We are able to also report a preliminary USNO optical parallax of $2 \mathrm{M} 2148+40$, which was classified as L6 in the optical and L6.5 peculiar in the near-infrared by its discovery paper (Looper et al. 2008) and is L6 FLD-G in the Allers \& Liu (2013a) system. The observed absolute parallax is $124.07 \pm 0.55$ mas,

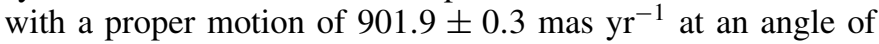
$59.1 \pm 0.1$ (Table 2). The distance of $8.060 \pm 0.036 \mathrm{pc}$ places it just outside the " 8 pc" sample. The radial velocity is unknown, 


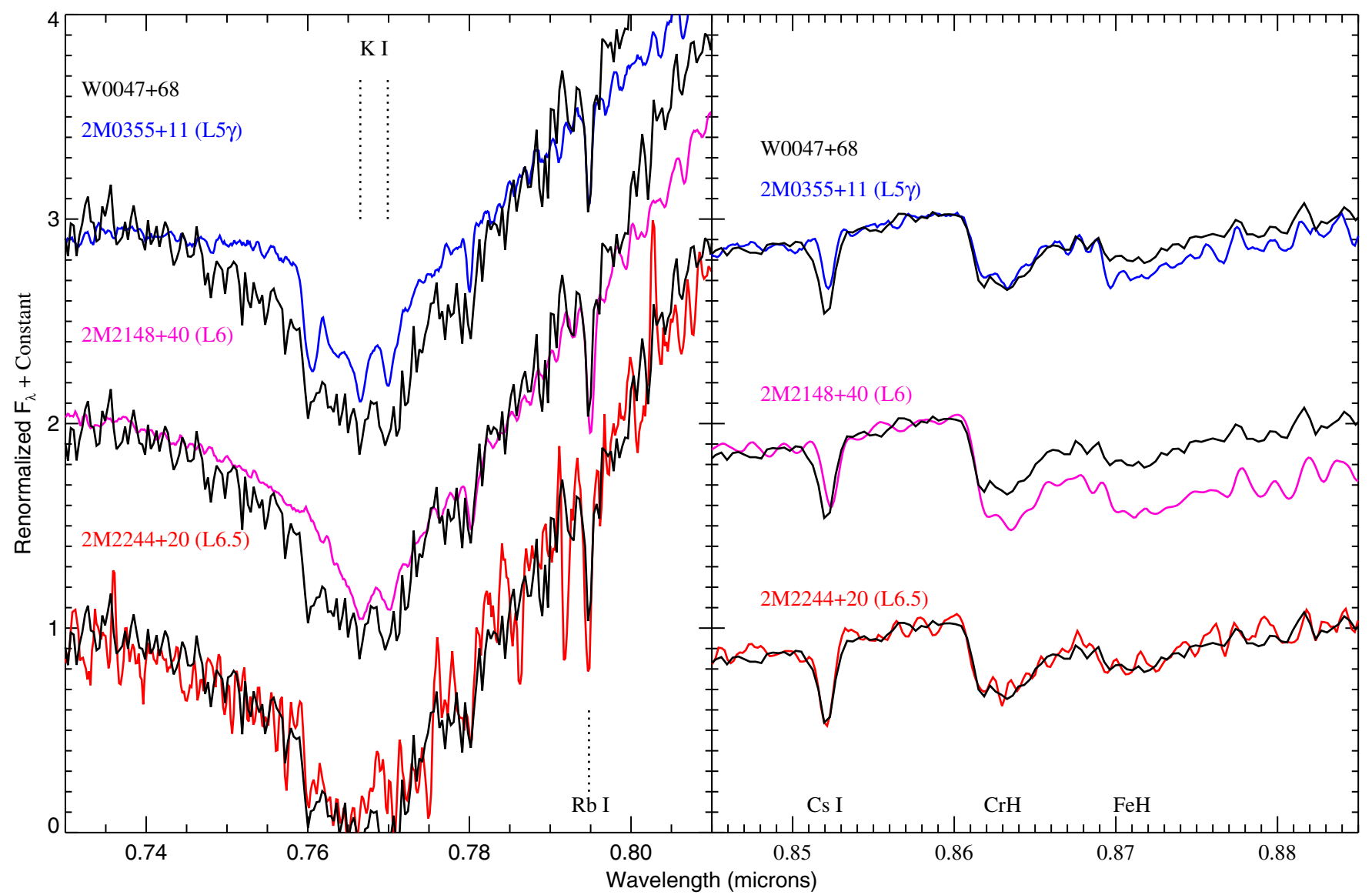

Figure 5. Same as Figure 4, but for the unusually red L dwarfs 2M0355+11 (Cruz et al. 2009), 2M2148+40 (Looper et al. 2008) and 2M2244+20 (Kirkpatrick et al. 2008). W0047+68 appears later/cooler than $2 \mathrm{M} 0355+11$ and $2 \mathrm{M} 2148+40$, but has a nearly identical spectrum to $2 \mathrm{M} 2244+20$. The Cs I are well-matched in $2 \mathrm{M} 2244+20$ and W0047+68, but the signal-to-noise is not sufficient to compare the Rb I line. Again, the spectra are from different instruments so we have smoothed the data to match resolutions.

but because of the position on the sky $(l=89.0, b=-10.5)$, this mainly affects the $V$ component of space motion. $U$ is strongly constrained: if we adopt $v_{\text {rad }}=0 \pm 40 \mathrm{~km} \mathrm{~s}^{-1}$, then $U=-34.0 \pm 0.5 \mathrm{~km} \mathrm{~s}^{-1}$, and $W=-5.6 \pm 7 \mathrm{~km} \mathrm{~s}^{-1}$. This rules out membership in any of the known very young nearby moving groups. $2 \mathrm{M} 2148+49$ is intrinsically fainter at the $J$ band than ordinary field L6 dwarfs and brighter at WISE bands.

\subsection{Photometry}

W0047+68 was observed as part of normal survey operations of the Pan-STARRS wide-field optical/NIR PS1 survey (Kaiser et al. 2010). We report AB magnitudes for the $y$ and $z$ bands, which are described in Tonry et al. (2012). The source was not detected in the shorter wavelength bands. WISE and Two Micron Sky Survey (2MASS) photometry were previously reported in Paper I.

We monitored W0047+68 in the $J$ band using the FLAMINGOS camera (Elston et al. 2003) on the Kitt Peak $2.1 \mathrm{~m}$ telescope in 2012 October (NOAO-Program 12B-0233) on three consecutive nights for four hours per night, after the main target for the program had set. We find no evidence of variability at the level of 0.05 mag or greater.

Although unobservable with WISE, many of the directly imaged planets have been observed at $L^{\prime}$ and $M^{\prime}$. W0047+68 has not been observed through these filters, but we may estimate the magnitudes using WISE photometry and other L dwarfs. Leggett et al. (2007) measured $M^{\prime}=11.90 \pm 0.03$ for $2 \mathrm{M} 2244+20$.
Scaling by the $W 2$ magnitudes, this suggests $M^{\prime}=11.04$ for W0047+68 and $M_{M^{\prime}}=10.6$.

\subsection{Luminosity}

We can obtain the bolometric magnitude of W0047+68 by integrating the spectral energy distribution, improving the estimate from Paper I using our new data. The near-infrared component is well-constrained by the Paper I spectrum normalized to agree with the 2MASS photometry. In the optical, we use the MMT spectrum. There is negligible contribution at less than $0.6 \mu \mathrm{m}$. We use the Spitzer Infrared Spectrograph (IRS) spectrum of 2M2244+20 (Stephens et al. 2009) renormalized by a factor of 2.23 (from the relative WISE $W 1$ and $W 2$ photometry) in the range of $5.15 \mu \mathrm{m}-14.15 \mu \mathrm{m}$ and a Rayleigh-Jeans tail beyond that. This spectral energy distribution is shown in Figure 6. The wavelength range between $2.55 \mu \mathrm{m}$ and $5.15 \mu \mathrm{m}$ is constrained by the WISE photometry, but we require higher resolution to account for the non-smooth spectrum. We consider the A-type (1200 K) and AE-type (1300-1500 K) models of Madhusudhan et al. (2011) and the preliminary BT-Settl $(1300-1500 \mathrm{~K})$ of Allard et al. (2013). In each case, we integrate the model spectra over the range of $2.55-4.00 \mu \mathrm{m}$, normalized to the WISE $W 1$ photometry, and the range of $4.00-5.15 \mu \mathrm{m}$, normalized to the WISE W2 photometry. (Because the observed colors are redder than the models, we allow discontinuities.) Averaging the three best-fitting near-infrared models (A $1200 \mathrm{~K}, \mathrm{AE}$ $1300 \mathrm{~K}$, and BT-Settl $1400 \mathrm{~K}$, all $\log g=4.0$ ) we obtain an 


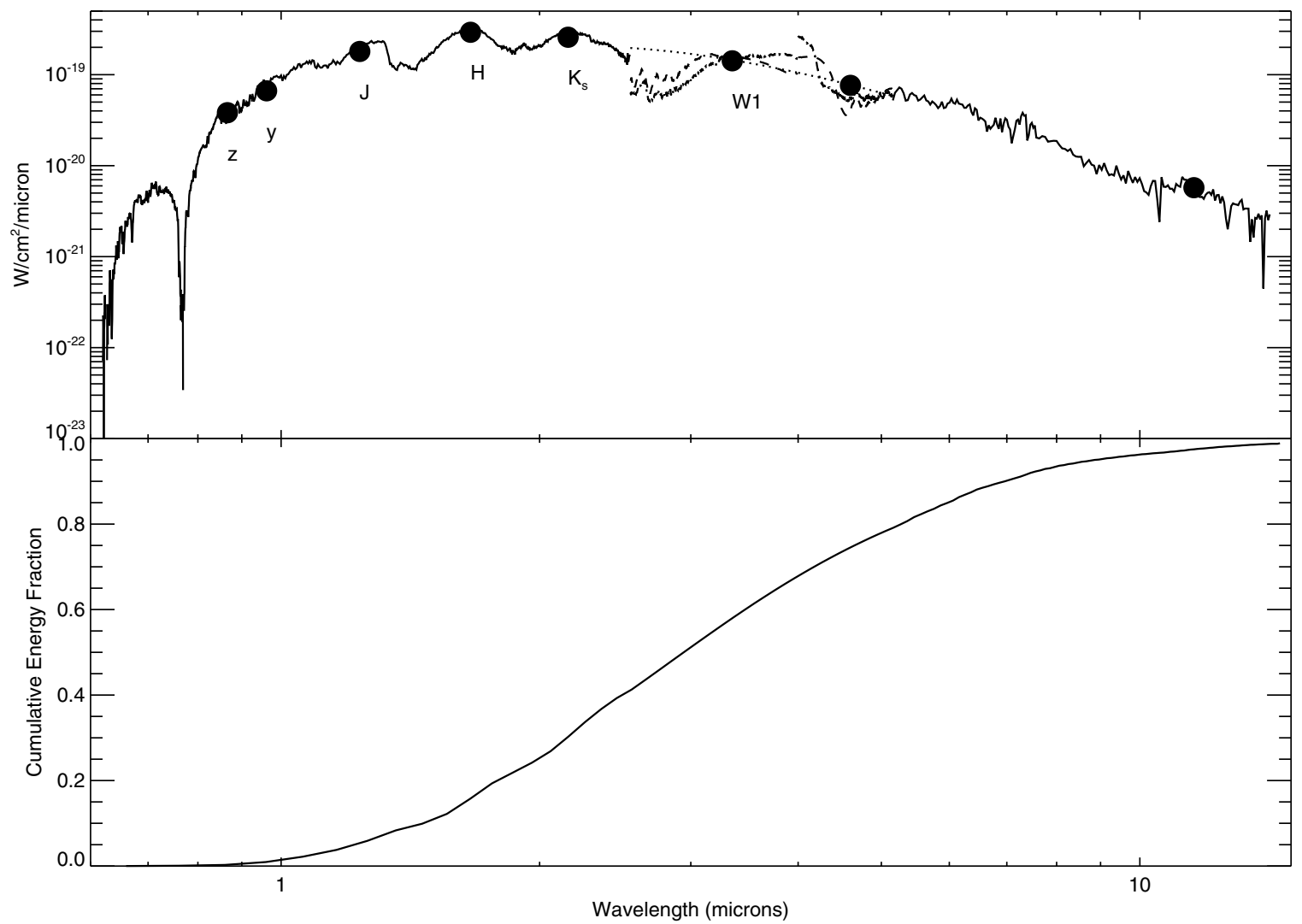

Figure 6. Top: the observed spectral energy distribution. The Pan-STARRS, 2MASS, and WISE photometry shown as solid circles. The solid lines show the optical (MMT) and near-infrared (IRTF) spectra of W0047+68 and the Spitzer IRS spectrum of 2M2244 normalized to match W0047+68. The dotted lines show a very thick cloud (A-type) model (Madhusudhan et al. 2011) with $T_{\text {eff }}=1200 \mathrm{~K}, \log g=4.0$, a BT-Settl model (Allard et al. 2013) with $T_{\text {eff }}=1400 \mathrm{~K}, \log g=4.0$, and a blackbody $(1400 \mathrm{~K})$ spectra normalized to match the $W 1$ and $W 2$ photometry; these are allowed to have discontinuities at $2.55 \mu \mathrm{m}, 4.00 \mu \mathrm{m}$, and $5.15 \mu \mathrm{m}$. Bottom: the cumulative spectral energy fraction. Half of the total energy of W0047+68 is emitted shortward of $2.9 \mu \mathrm{m}$.

apparent bolometric magnitude of 16.30 with an uncertainty of \pm 0.02 magnitudes due to the spread in models, all of which feature deep water bands. If blackbodies without absorption bands are used for this region, the resulting bolometric magnitude is up to 0.05 mag brighter, while an observed $A K A R I$ spectrum of an ordinary L8 dwarf (Sorahana \& Yamamura 2012; Sorahana et al. 2013) normalized to the $W l$ photometry is 0.02 mag fainter; other, higher-gravity bluer models are within this range of uncertainty. There is an additional uncertainty from the uncertainty in the 2MASS and WISE photometry and other spectra, which we estimate is $\pm 0.025 \mathrm{mag}$. The derived W0047+68 luminosity is $(3.58 \pm 0.29) \times 10^{-5} L_{\odot}$. The cumulative contribution to the luminosity is also shown in Figure 6: half of the energy is emitted redward of $2.9 \mu \mathrm{m}$. Following the same procedure for $2 \mathrm{M} 2148+40$, but with the BT-Settl $1500 \mathrm{~K}$ model yields an apparent bolometric magnitude of $15.12 \pm 0.03$ and luminosity of $L=(4.64 \pm 0.14) \times 10^{-5} L_{\odot}$.

\section{PROPERTIES OF W0047+68}

\subsection{AB Dor Moving Group Membership}

The spectra of W0047+68 include a number of age indicators. The most conservative age estimate for W0047+68 comes from the fact that we detect lithium: for the observed luminosity, this indicates that its age is less than one billion years old (Chabrier et al. 2000). The near-infrared spectroscopy, however, points to an even younger age, less than that of most lithium L-type brown dwarfs. Thompson et al. (2013) suggested that W0047+68 is a "low-gravity object and hence young," based on their analysis of a moderate-resolution spectrum similar to the one we have obtained. Our near-infrared surface gravity classification of INT-G (Section 2.1) indicates an age of $\sim 100-150 \mathrm{Myr}$ for W0047+68, but not $<50 \mathrm{Myr}$, though this system is primarily based upon warmer objects. Many of the youngest stars are found to be members of moving groups.

The three-dimensional space velocity (Figure 7) is consistent with the young AB Dor Moving Group (Zuckerman et al. 2004; Torres et al. 2008). ${ }^{13}$ Most ABDMG members are in the southern hemisphere, but in fact the group is quite loose and includes northern hemisphere members. Given the parallax, proper motion, radial velocity, and lithium age constraint, we find that the BANYAN II model (Gagné et al. 2013) assigns an ABDMG membership probability of $99.96 \%$ to W0047+68. As seen in Figure 7, W0047+68 lies fairly close to the group in physical space, and the peculiar velocity necessary to move W0047+68 from the nucleus of the ABDMG to its present position is $<1 \mathrm{~km} \mathrm{~s}^{-1}$. Furthermore, as discussed in detail in Section 3.5, mid-L dwarf ABDMG candidate members, including the companion CD-35 2722B (Wahhaj et al. 2011) which is a definite member, are unusually red in $J-K$ color.

Therefore, on the basis of three indicators (three-dimensional space motion, spectroscopic surface gravity, and near-infrared color), we propose that W0047+68 is a bona fide member of the ABDMG. One could ask if it is plausible that an ABDMG brown dwarf member is so close to the Sun. Torres et al. (2008)

\footnotetext{
13 In Paper I, we noted that the direction of the proper motion of W0047+68 agreed with the very young $(\sim 12 \mathrm{Myr}) \beta$ Pic Moving Group. The parallax rules $\beta$ Pic Moving Group membership out.
} 

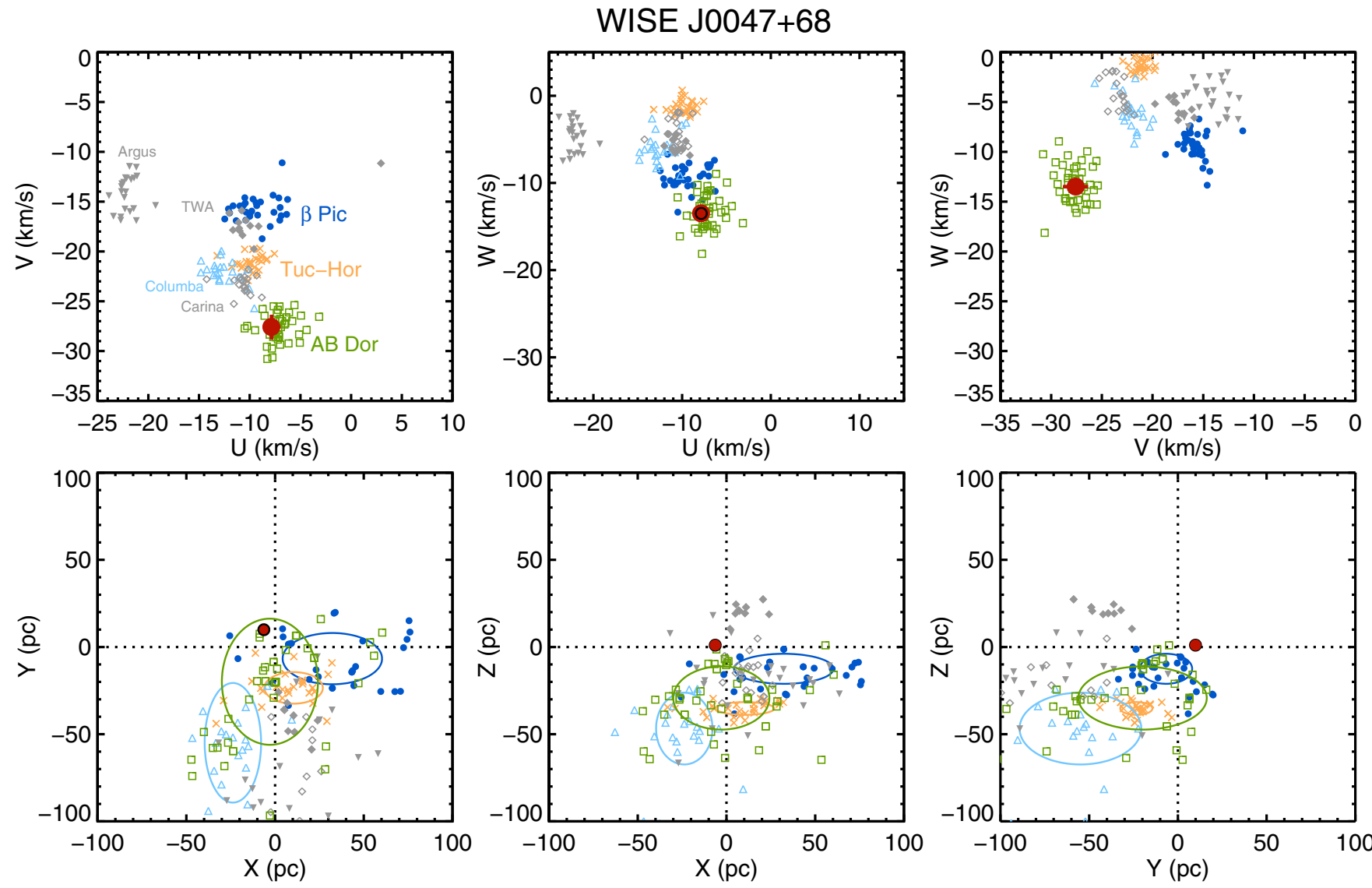

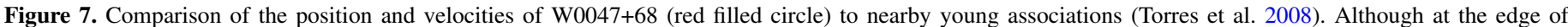

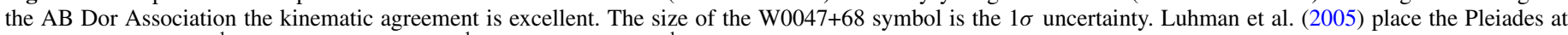

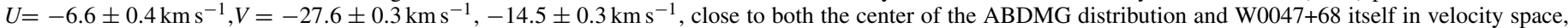
but in physical space the cluster is located at $X=-119 \mathrm{pc}, Y=28 \mathrm{pc}$, and $Z=-53 \mathrm{pc}$.

list eight ABDMG M dwarf members within $16 \mathrm{pc}$ of the Sun and Kirkpatrick et al. (2012) find that the ratio of brown dwarfs to stars is $1: 6$, so the presence of one or two nearby ABDMG brown dwarfs is not surprising.

\subsection{Age and Metallicity for W0047+68}

We can leverage the current knowledge of the ABDMG, which is based on a wide variety of stellar masses and spectral types, to understand the properties of W0047+68 and the origin of its peculiar spectrum. Although early discussions of ABDMG (Zuckerman et al. 2004) assumed an age of $\sim 50$ million years, current evidence points to an age of $\sim 100-125 \mathrm{Myr}$ (Luhman et al. 2005; Barenfeld et al. 2013). Indeed, Luhman et al. (2005) argue that ABDMG is "roughly co-eval" with the Pleiades and is physically related given the similar space velocities, an argument supported by Ortega et al. (2007). This age is in excellent agreement with our spectroscopic estimate of surface gravity intermediate between the youngest association and older field brown dwarfs. Of course, even the age of the rich, wellstudied Pleiades is still somewhat uncertain (see Soderblom et al. 2009), but W0047+68's age constraint is much better than that of most known brown dwarfs.

The relative importance of surface gravity and composition is debated in both unusually red brown dwarfs and massive gas giant planets. Crucially, the composition of the ABDMG is also known to be near solar. Ortega et al. (2007) compiled the recent literature measurement of 15 ABDMG members and found a mean $[\mathrm{Fe} / \mathrm{H}]=-0.02 \pm 0.02$. Viana Almeida et al.
(2009) measured a mean $[\mathrm{Fe} / \mathrm{H}]=-0.01]$ for $12 \mathrm{ABDMG}$ members, with a standard deviation of 0.09 , but after applying a correction for a trend in effective temperature found a mean $[\mathrm{Fe} / \mathrm{H}]=0.04$ with a standard deviation of 0.05 . They also reported that for their entire young star sample the mean values are $[\mathrm{Si} / \mathrm{H}]=-0.11$ and $[\mathrm{Ni} / \mathrm{H}]=0.01$. Biazzo et al. (2012) reported a mean $[\mathrm{Fe} / \mathrm{H}]=0.10 \pm 0.03$ for the five stars in the ABDMG, and found that 10 elements ( $\mathrm{Na}, \mathrm{Mg}, \mathrm{Al}, \mathrm{Si}, \mathrm{Ca}$, $\mathrm{Ti}, \mathrm{Cr}, \mathrm{Fe}, \mathrm{Ni}$, and $\mathrm{Zn}$ ) have abundance patterns that are solar and "typical" of the local Galactic thin disk. Barenfeld et al. (2013) claim a somewhat lower $[\mathrm{Fe} / \mathrm{H}]=0.02 \pm 0.02$ for the ABDMG nucleus and measure seven other elements $(\mathrm{Na}$, $\mathrm{Mg}, \mathrm{Al}, \mathrm{Si}, \mathrm{Cr}, \mathrm{Mn}$, and $\mathrm{Ni}$ ) to be close to solar values. Since these $[\mathrm{Fe} / \mathrm{H}]$ values are characteristic of the bulk of the local thin disk, the vast majority of field L dwarfs must have similar values. Whether the scatter in values is a matter of observational uncertainties or true scatter could be debated. Barenfeld et al. (2013) argue that they see real scatter in composition in the ABDMG "stream" members and that, therefore, many of the more distant ABDMG objects are not from a common origin. Nevertheless, even these objects are all near solar in $[\mathrm{Fe} / \mathrm{H}]$ and other elements, as is the Pleiades (Soderblom et al. 2009), so we conclude that W0047+68's composition is constrained to be near-solar even if the ABDMG stream is not truly homogeneous. This constraint includes the key elements involved in cloud formation: $\mathrm{Fe}$ and $\mathrm{Al}$ (along with $\mathrm{O}$ ) are the major elements for high-temperature condensates (i.e., in the deeper atmospheric layers of $\mathrm{W} 0047+68$ ) and $\mathrm{Mg}$ and $\mathrm{Si}$ (and O) at moderate 


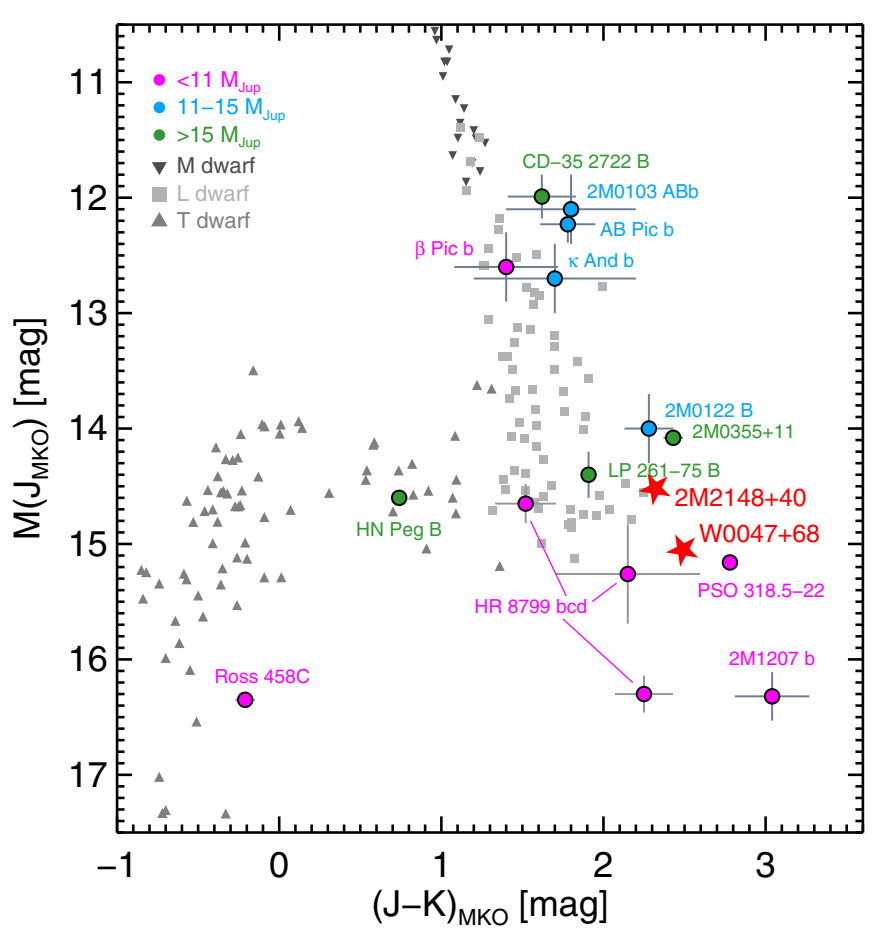

Figure 8. Color-magnitude diagram comparing W0047+68 and 2M2148+40 to ordinary field L and T dwarfs, very low mass brown dwarfs, and exoplanets compiled by Liu et al. (2013b).

temperatures characteristic of the upper atmospheric layers (Marley et al. 2002; Helling et al. 2008b; Visscher et al. 2010).

Using the Chabrier et al. (2000) models and the observed luminosity, W0047+68 would have $\log g \approx 4.5$ and $M \approx$ $0.018 M_{\odot}$ for age $120 \mathrm{Myr}$. Similar values are found with the Burrows et al. (1997) models. The low surface gravity and solar metallicity suggest that the thick clouds of W0047+68 are a normal feature of a young brown dwarf. In Figure 8, we show the color-magnitude diagram for brown dwarfs and planetary mass objects (Liu et al. 2013b), updated with W0047+68 and $2 \mathrm{M} 2148+40$. W0047+68 lies close to PSO J318-22, adding to the evidence of a low-gravity sequence that extends redward of the normal L/T brown dwarf sequence.

\subsection{Effective Temperature}

In Paper I, we found that although models from different groups agreed that unusually thick clouds were needed to fit the near-infrared spectrum, the predicted effective temperatures ranged from as high as $1600 \mathrm{~K}$ to as low as $1100 \mathrm{~K}$. With our measurement of the luminosity, we can make another estimate of the effective temperature using theoretical predictions of the radius. We find

$$
T_{\text {eff }}=(1413 \mathrm{~K} \pm 28 \mathrm{~K})\left(\frac{0.1 R_{\odot}}{R}\right)^{0.5} .
$$

The highest temperature and smallest radius for W0047+68 can be obtained by using the oldest evolutionary models in which lithium has not been depleted. This would allow $T_{\text {eff }} \approx 1450 \mathrm{~K}$ and an age of $\sim 1$ billion years, with $\log g \approx 5$. ABDMG membership implies a larger radius and lower temperature. Adopting an age of $125 \mathrm{Myr}$ for ABDMG membership, Burrows et al. (1997) have $M_{\odot}=0.020 M_{\odot}$ and $R=0.117 R_{\odot}$; Chabrier et al. (2000) have $M=0.018 M_{\odot}$ and $R=0.124 R_{\odot}$. Correspondingly, our best estimate of W0047+68's effective temperature is $T_{\text {eff }} \approx 1270-1300 \mathrm{~K}$ with $\log g \approx 4.5$. Burrows et al. (2011) report that high metallicity or thick cloud models each lead to an increase in the radius over "standard" models, which would lower the temperature below $1400 \mathrm{~K}$ even if W0047+68 is old.

Given an effective temperature of $\sim 1300 \mathrm{~K}$, W0047+68 shows that intermediate surface gravity brown dwarfs have a much different L/T-type transition. For older, high gravity field brown dwarfs, this effective temperature is normally associated with the early T dwarfs (Cushing et al. 2008; Stephens et al. 2009) and certainly with spectral types that have already begun the move to the blue in $J-K$. The early T dwarfs have methane bands in the $H$ band and $K$ band (Burgasser et al. 2006). No such features are seen in W0047+68, and we also did not detect the high amplitude $J$-band variations seen in $\sim 1300 \mathrm{~K}$ early T dwarfs and attributed to patchy clouds (Artigau et al. 2009; Radigan et al. 2012; Apai et al. 2013). (However, W0047+68's low $v \sin i$ suggests that we may be viewing it near pole-on.) We emphasize that this result depends upon the evolutionary models: W0047+68 has a luminosity typical of that of ordinary L7 dwarfs (Vrba et al. 2004; Golimowski et al. 2004), but its young age (and theoretically larger radius) requires a lower temperature. As shown by Liu et al. (2013b), the younger object PSO J318-22 is an even more extreme case, with nearly the same luminosity and spectral type as W0047+68, but a deduced temperature of $1160_{-40}^{+30} \mathrm{~K}$.

\subsection{Comparison to Thick Cloud Models}

The properties of condensate cloud layers depend on complex processes that are difficult to model completely, including nucleation, growth, and settling (Ackerman \& Marley 2001; Helling et al. 2008b). Each theoretical effort to predict synthetic spectra makes different choices in parameterizing the cloud properties. These were compared by Helling et al. (2008a) in test cases aimed at modeling typical field brown dwarfs. In Paper I, we discussed how theoretical models from three different groups (UCM; Tsuji 2005; $f_{\text {sed }}$, Ackerman \& Marley 2001; Marley et al. 2002; Stephens et al. 2009; very thick (A-type) and intermediate thickness (AE-type) cloud models, Madhusudhan et al. 2011) were able to fit the low-resolution W0047+68 near-infrared spectrum. In each case, models can fit the $J-K$ color and qualitatively reproduce the spectrum by adjusting a free parameter to increase the thickness of the condensate clouds. Another set of models, DRIFT-PHOENIX (Witte et al. 2009), was used by Witte et al. (2011) to fit low-resolution near-infrared spectra of $2 \mathrm{M} 2148+40$ and $2 \mathrm{M} 2244+20$. They derived warm temperatures $\left(T_{\text {eff }}=1500 \mathrm{~K}\right)$ and very low gravities ( $\log g=3.0$ and 3.5, respectively), but discussed the model grid limitations for such red objects and concluded that their temperatures are likely overestimated and the gravities underestimated.

We can now also compare to a fifth set of models. Allard et al. $(2012,2013)$ have presented a new model family called BT-Settl. The mixing and cloud properties are informed by two-dimensional radiation hydrodynamic (hereafter RHD) simulations (Freytag et al. 2010). The result is that for cool L dwarfs, low-gravity objects are predicted to be redder, with thicker clouds. We show the W0047+68 observed spectrum with the model predictions in Figure 9. While our analysis of W0047+68 favors $T_{\text {eff }} \approx 1300 \mathrm{~K}$ and $\log g \approx 4.5$, the BT-Settle grid requires an even lower gravity but higher temperature $\left(T_{\text {eff }}=1400-1500 \mathrm{~K}, \log g=4.0\right)$ to match the spectrum. 


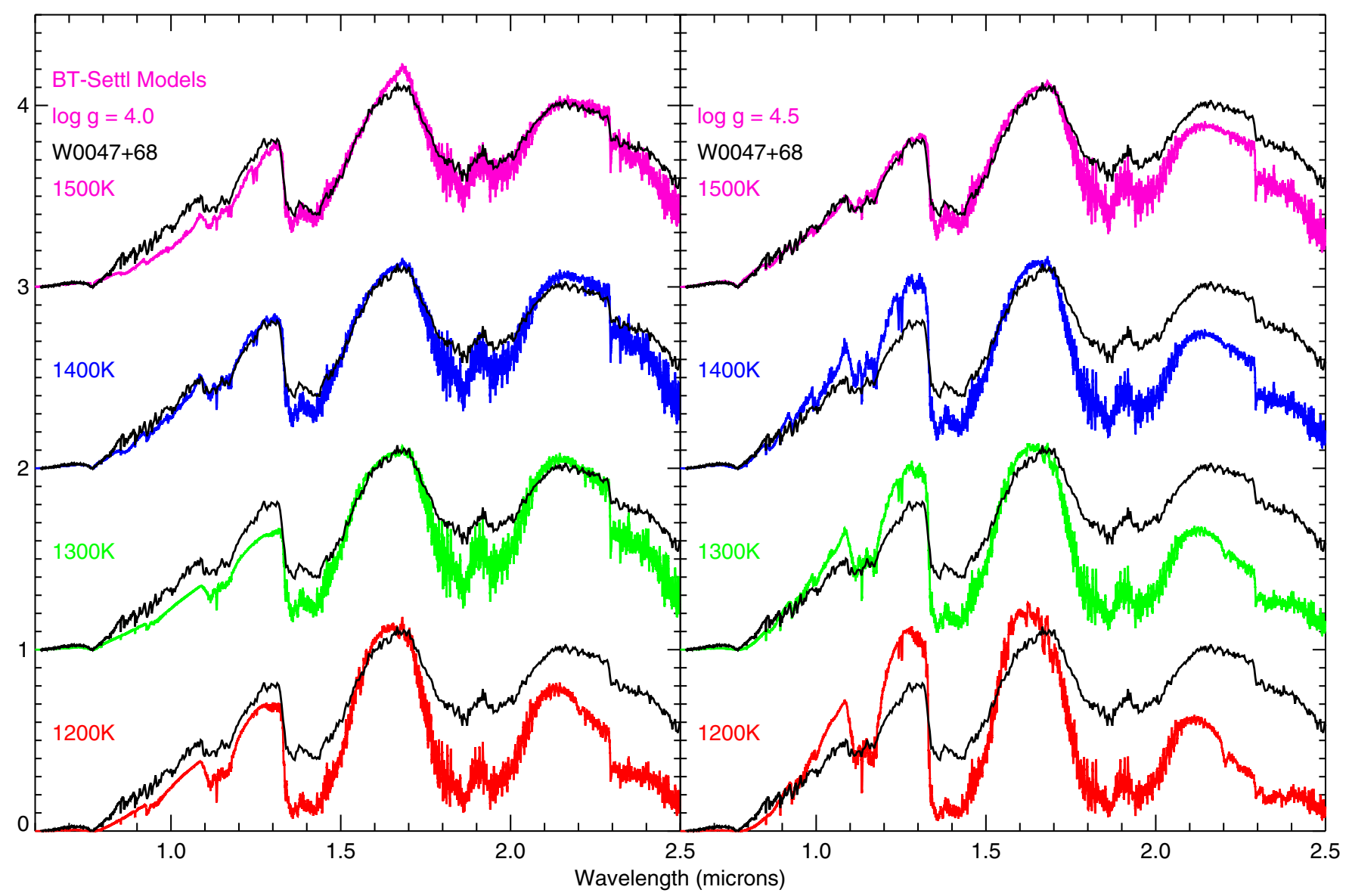

Figure 9. Comparison of observed W0047+68 spectrum to low-gravity ( $\log g=4.0,4.5)$ BT-Settl models: each is normalized to agree in the $1.50-1.75 \mu \mathrm{m}$ region. While we conclude that $T_{\text {eff }}=1300-1430 \mathrm{~K}$ and $\log g=4.5$, the models with $\log g=4.0$ do a better job of reproducing the red near-infrared spectrum.

(Note that the $\log g=3.5$ BT-Settl models are even redder and can also fit.)

It has long been recognized that $J-K$ colors are relatively poor temperature indicators for brown dwarfs, even among the typical field population because of the effects of clouds (Marley et al. 2002; Knapp et al. 2004; Stephens et al. 2009). Our results again underline this issue. The Unified Cloudy Model and BT-Settl give qualitatively good fits to W0047+68, but are warmer by $100-200 \mathrm{~K}$ than our estimate of $1300 \mathrm{~K}$, while, on the other hand, the very thick (A-type) and $f_{\text {sed }}=1$ models give temperatures of $100-200 \mathrm{~K}$ too low when matching the spectra. The plots in Figure 9 have arbitrary normalizations. An interesting check is to derive the radius from the near-infrared region alone instead of using our procedure in Section 3.3: given a model, the observed parallax, and the observed flux-calibrated spectrum, the only adjustable parameter is the radius. The BT-Settl results when fitting over the range of 1.2-2.5 $\mu \mathrm{m}$ with the $\log g=4.0$ models are $0.13 R_{\odot}(1200 \mathrm{~K}), 0.12 R_{\odot}(1300 \mathrm{~K})$, $0.94 R_{\odot}(1400 \mathrm{~K})$, and $0.82 R_{\odot}(1500 \mathrm{~K})$, consistent with the discussion in Section 3.3. The $1500 \mathrm{~K}$ model has a qualitatively good fit but requires a radius that is considerably smaller than we expect for a brown dwarf with lithium. We believe the balance of evidence is against this possibility, though it should be noted that Sorahana et al. (2013) have used their own model fitting to AKARI data to argue that (ordinary) field late-L dwarfs are warmer and much smaller than predicted. Cushing et al. (2008) showed that fits to limited wavelength regions can almost always work, but can give much different effective temperatures than fits to the entire spectral energy distribution.
One important conclusion from our work is that model colors should not be used to derive surface gravities because they cannot yet predict cloud properties well enough. In the cases with a full grid of surface gravities (Madhusudhan et al. 2011; Allard et al. 2012), one must go to $\log g=4.0$ or even less to get thick enough clouds to match in the infrared. This would in turn require that W0047+68 be below the deuterium-burning limit and very young, much younger than the best estimates of the ABDMG age. For example, in the Baraffe et al. (2002) models at $20 \mathrm{Myr}$, a $0.007 M_{\odot}$ would have $\log g=4.0$ and match the observed luminosity with $T_{\text {eff }}=1200 \mathrm{~K}$, while a $0.009 M_{\odot}$ would have $\log g=4.1$ and $T_{\text {eff }}=1400 \mathrm{~K}$ but would be $90 \%$ more luminous than observed. Manjavacas et al. (2014) have argued that the high altitude dust in BT-Settl models should be increased to better fit the warmer (M9.5-L3) young brown dwarfs; the same is evidently true for W0047+68.

The optical spectrum of W0047+68 also poses a challenge for our understanding. The spectrum is dominated by the broad wings of the $\mathrm{K}$ I which, in turn, are the dominant feature that can be used for spectral typing; these are evidently saturated around $1300 \mathrm{~K}$ even though their weakness is an indicator of low gravity in warmer objects (Cruz et al. 2009). This was recognized by Kirkpatrick et al. (2008) who argued that $2 \mathrm{M} 2244+20$ is young, yet found a normal $\mathrm{L}$ dwarf spectrum (shown in our Figures 3 and 5). They speculated that "the lower gravity (optical) spectrum will have weaker alkalis and stronger hydrides than a high-gravity L dwarf of comparable temperature." This, in turn, creates "a degeneracy at mid- and late-L-types whereby low-gravity objects are very difficult or 


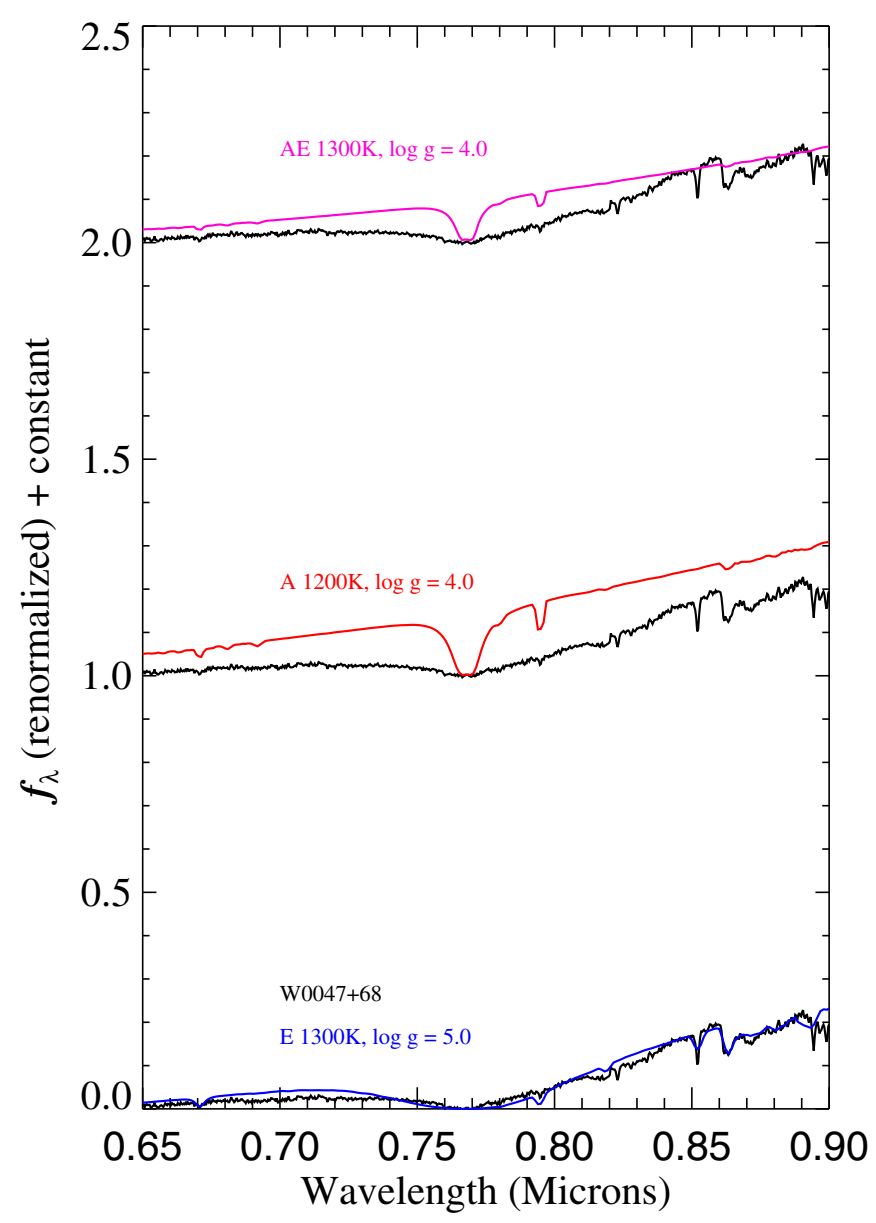

Figure 10. Comparison of observed W0047+68 spectrum to Madhusudhan et al. (2011) intermediate thickness clouds (AE type) and very thick cloud (A type) models with low surface gravity $(\log g=4.0)$ models. These two models are normalized to the W0047 $\mathrm{H}$-band region (as in Paper I). The strength of the broad potassium line is under-predicted, and the pseudo-continuum is too high, due to the overly low surface gravity. We also show a Burrows et al. (2006) model with $1300 \mathrm{~K} \log g=5.0$ and normal, thin ("E-type") clouds, normalized in the region $0.7-0.9 \mu \mathrm{m}$, which demonstrates that this model family does reproduce the main features of $\mathrm{L}$ dwarfs in the optical.

impossible to distinguish in the optical." If our interpretation of W0047+68 is correct, this must be the case because (except for the strengths of the $\mathrm{Cs}$ and $\mathrm{Rb}$ lines) it is very close to the L7 standard. Another important factor in creating degeneracy may be additional opacity due to dust, which we know must be different in the near-infrared and may be different in the optical. Pavlenko et al. (2000) discussed the optical spectra of L dwarfs and showed that the broad $\mathrm{K}_{\mathrm{I}}$ line and the sharper Cs I and $\mathrm{Rb}$ I lines in the $T_{\text {eff }}=1200-1500 \mathrm{~K}$ range are sensitive to surface gravity and dust extinction; they could change the best-fit effective temperature and surface gravity by increasing or decreasing them together, and additional opacity helped the quality of fits. Lodders (1999), Lodders \& Fegley (2002), and Marley et al. (2002) discuss the sensitivity of the K I chemistry to sedimentation and cloud formation. Thus, it may be possible to find models where the "L7" optical spectra are relatively insensitive to gravity.

Synthetic optical spectra are available for the BT-Settl and Madhusudhan et al. (2011) model families, and have not been tuned to match observations. In Figure 10, we compare the predicted and observed far-red spectra using the Madhusudhan et al. 2011 very-thick (A) and intermediate thickness (AE) models normalized to agree in the $H$ band (as in Figure 6 of Paper I). In both cases, the absorption by the broad $\mathrm{KI}$ wings is underestimated and, as a result, the continuum is too high. Since the optical and near-infrared spectra of typical L dwarfs are fit well by the "E type" thin cloud models and $\log g=5.0$ (Burrows et al. 2006), one would expect them to also fit the optical spectrum of W0047+68. Such a fit is also shown in Figure 10. The optical BT-Settl models are shown in Figure 11. There are also disagreements with the observations, with the pseudo-continuum lying too low, but the $T_{\text {eff }}=1300 \mathrm{~K}$, $\log g=4.0$ model is reasonably similar in overall morphology and slope, though it lacks the hydrides; $\mathrm{CrH}$ and $\mathrm{FeH}$ are better matched with the $\log g=4.5$ models. The change in shape of the core of the $\mathrm{K}$ I resonant doublet in the $\log g=4.0$ models from $1500 \mathrm{~K}$ to $1300 \mathrm{~K}$ is similar to the differences between $2 \mathrm{M} 0355+11$ and W0047+68 (Figure 5), and suggests that the basic picture in which the core of the $\mathrm{K}_{\mathrm{I}}$ feature is saturated for late L dwarfs even for low gravities may be correct. In both the BT-Settl and A/AE models, the $\log g=4.0$ models have no $\mathrm{CrH}$ or FeH features: this prediction could be tested in the $\beta$ Pic Moving Group planetary mass object PSO J318-22.

\subsection{AB Dor Moving Group Brown Dwarfs}

There are now a number of L-type ${ }^{14}$ brown dwarfs proposed as ABDMG members. The strongest members, CD-35 2722B (Wahhaj et al. 2011), 2M0355+11 (Faherty et al. 2013b; Liu et al. 2013a), and W0047+68, show a surprising spread in spectral properties. 1RXS J2351+3127 B (Bowler et al. 2012) and 2MASS 01222439 B (Bowler et al. 2013) still need kinematic confirmation. The similarity of $2 \mathrm{M} 2244+20$ and W0047+68 supports ABDMG membership for $2 \mathrm{M} 2244+20$; using the W0047+68 absolute magnitudes for 2M2244+20 implies a distance consistent with ABDMG tangential motion (Gagné et al. 2014). The signal-to-noise on the $2 \mathrm{M} 2244+20$ spectrum is too low for a reliable measurement of lithium. In Figure 12, we plot the intermediate-resolution near-infrared spectra of 1RXS J2351+3127 B, CD-35 2722B, 2MASS 01222439 B, 2M0355+11 (Liu et al. 2013a), and W0047+68.

Allers \& Liu (2013a) have discussed the discrepancies between 2M0355+11 (L3 VL-G) and CD-35 2722B, which is L3 INT-G and a companion to one of the nucleus AB Dor members. Most notably, 2M0355+11 is more strongly peaked in the $H$ band. Despite the very similar $J-K_{s}$ colors of W0047+68 and $2 \mathrm{M} 0355+11$, the surface gravity sensitive features are very different, and W0047+68 appears to be cooler. $2 \mathrm{M} 0355+11$ is also a magnitude brighter in the color-magnitude diagram (Figure 8). The Pleiades has a similar age and space motion, and infrared spectroscopy shows early L dwarfs in the Pleiades are unusually red (Bihain et al. 2010). Allers \& Liu (2013b) report that these spectra can be classified as VL-G or INT-G but show a spread in properties. Unfortunately, no late-type $L$ dwarfs in the Pleiades are yet known, but our adopted age of ABDMG implies that W0047+68-like members should be found in deep Pleiades proper motion searches. Together, the ABDMG moving group brown dwarfs form an empirical intermediate surface gravity $(\log g=4.5)$ sequence.

One puzzle is that $2 \mathrm{M} 0355+11$ has two very obvious indicators of low surface gravity - the very peculiar optical spectrum (L5 $\gamma$ ), a very sharply peaked $H$ band-while W0047+68 has more subtle features. The inconsistency of the $H$-band shape as a gravity indicator is discussed in detail by Allers \& Liu (2013a).

\footnotetext{
${ }^{14}$ Delorme et al. (2012) have also proposed CFBDSIR 2149-0403, an unusually red $\mathrm{T}$ dwarf, as a candidate planetary mass ABDMG member.
} 


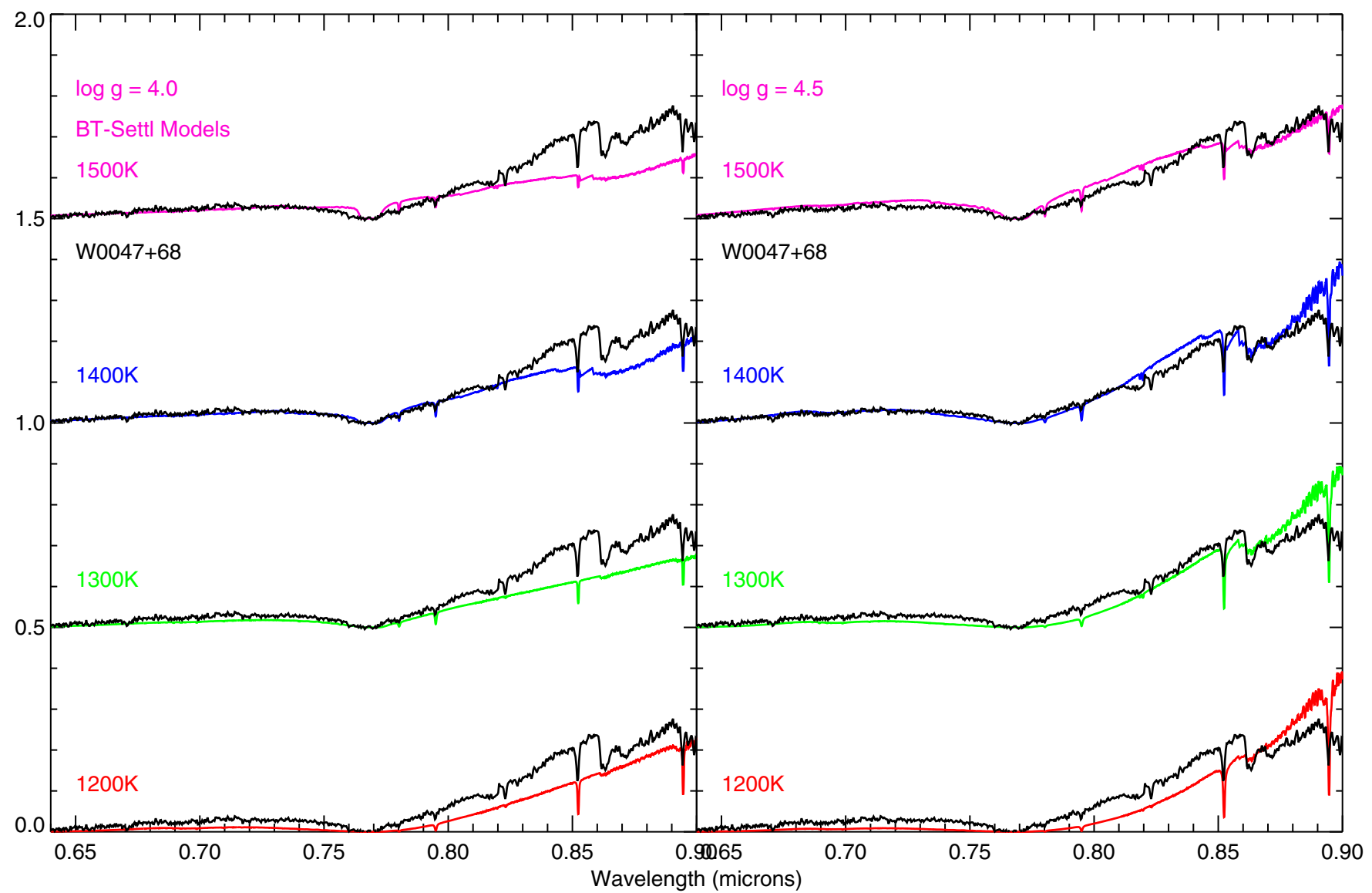

Figure 11. Comparison of observed W0047+68 spectrum to low-gravity ( $\log g=4.0,4.5)$ BT-Settl models: each is normalized to agree in the $1.5-1.75 \mu \mathrm{m}$ region. See the discussion in Section 3.4.
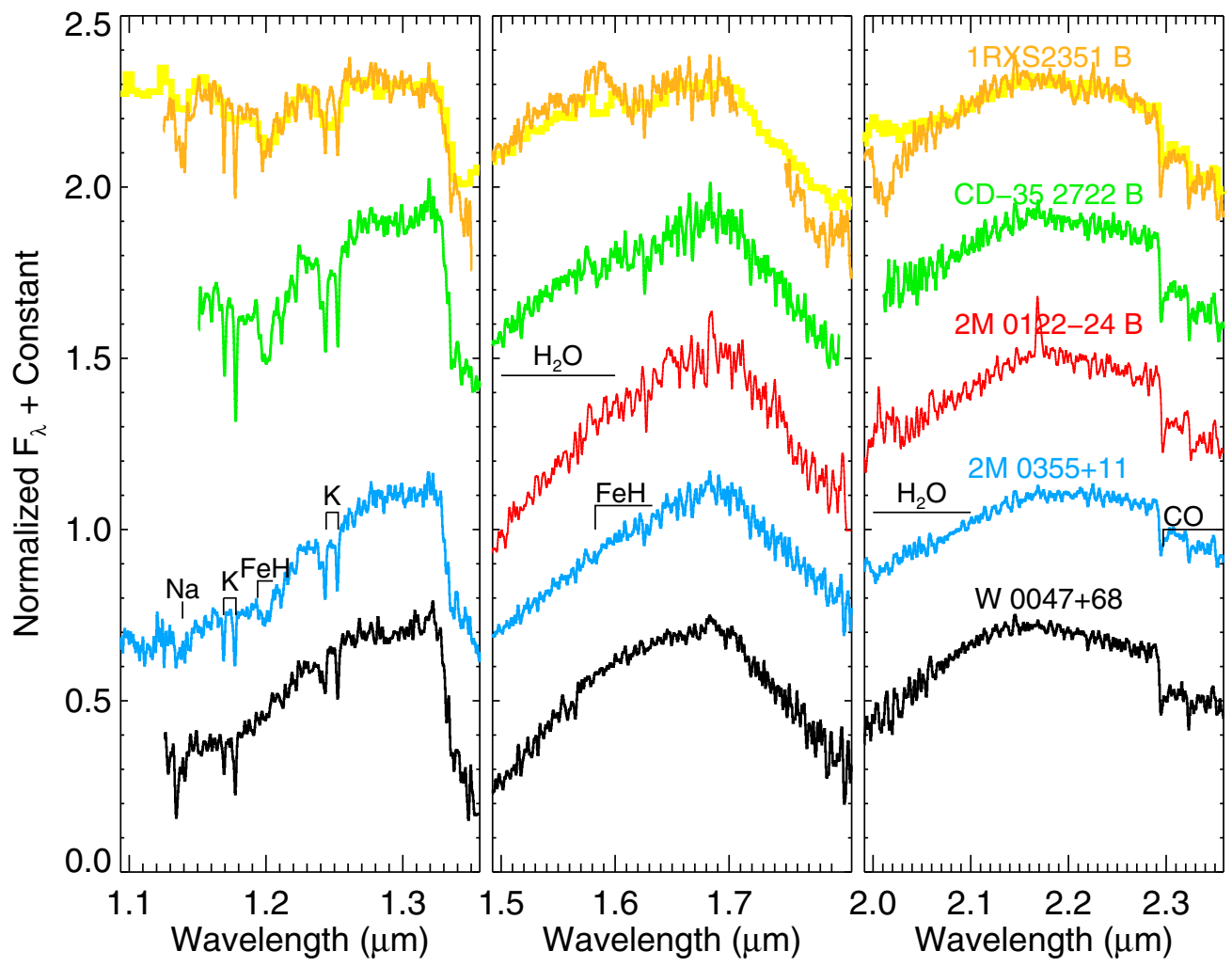

Figure 12. Comparison of ABDMG L-type brown dwarfs. Note the sharply peaked 2M0355+11 $\mathrm{H}$-band spectrum compared to the other cool L dwarf members. 
With regard to this pair of objects, three possibilities merit discussion. One possibility is that both $2 \mathrm{M} 0355+11$ and W0047+68 are normal ABDMG members whose differences are simply a consequence of temperature. Liu et al. (2013b) have found that the luminosity of $2 \mathrm{M} 0355+11$ is $\log L / L_{\odot}=-4.23 \pm 0.11$, i.e., $\sim 60 \%$ more luminous than W0047+68. This suggests that it is $\sim 170 \mathrm{~K}$ warmer than W0047+68, consistent with the two spectral types' earlier classification in the optical. The optical observed differences in the $\mathrm{K}_{\mathrm{I}}$ core (Figure 5) appear qualitatively similarly to the BT-Settl offsets between the $1500 \mathrm{~K} /$ $1400 \mathrm{~K}$ models and the $1300 \mathrm{~K}$ models for $\log g=4.0$; the BT-Settl low-gravity models are most "peaky" at $1500 \mathrm{~K}$, but the $H$ band becomes more rounded for lower temperatures. The A/AE models are also not "peaky" at the $H$ band for $1300 \mathrm{~K}$. A less likely possibility is both $2 \mathrm{M} 0355+11$ and W0047+68 are ABDMG members, but $2 \mathrm{M} 0355+11$ is actually a lower mass object very close to the deuterium-burning limit, resulting in a higher luminosity and temperature than W0047+68, but a lower surface gravity. This kind of complex behavior near the deuterium-burning limit at ages up to $\sim 120 \mathrm{Myr}$ appear in all published model tracks and have recently been discussed again theoretically by Spiegel et al. (2011) and observationally by Allers et al. (2010) and Bowler et al. (2013). Whether or not this suggestion matches in detail depends on the age of ABDMG and the exact behavior of model tracks near the deuteriumburning limit. The third possibility is that either, or both, are not ABDMG members. Of course, if $2 \mathrm{M} 0355+11$ has a lower gravity then it is a younger interloper, perhaps $\sim 30-50$ Myr old. This may not be as unlikely as it seems. Pre-Hipparcos work identified a "Local Association" (also known as the "Pleiades Super-Cluster" or "Pleiades Moving Group") including many young stars in the age range of 50-150 Myr (Eggen 1992; Jeffries 1995), with a dispersion of $\pm 10 \mathrm{~km} \mathrm{~s}^{-1}$. The ABDMG is part of this broader association. Montes et al. (2001) identified many young stars with Local Association motions, and found kinematic "substructure" as well as a fairly large spread in velocities. Makarov (2003) confirmed the Local Association streaming motion in an X-ray selected sample. Antoja et al. (2008; see their Figure 13) find many young ( $<100$ Myr) stars concentrated in this broad region of $U-V$ space. More controversially, López-Santiago et al. (2006) argued that the AB Dor group could be divided into a 30-50 Myr-old subgroup and a 80-120 subgroup. (There are also, of course, old stars with similar motion so kinematics alone cannot prove youth. In a non-age-selected Hipparcos sample, Bovy et al. 2009 find that $\sim 10 \%$ of stars can be associated with a Pleiades moving group.)

\section{2M2148+40: AN OLDER DUSTY L DWARF}

In Paper I, we remarked that in our low-resolution nearinfrared spectrum W0047+68 resembled a slightly cooler (or one subclass later) version of 2M2148+40. Looper et al. (2008) discovered this brown dwarf and argued that spectroscopic and kinematic evidence suggests it is not young, but is rather an older brown dwarf whose spectral peculiarities might be explained by high metallicity. (However, they did find 2M2148+40 has lithium with an equivalent width of $12.1 \pm 0.6 \AA$, see Figure 3.) At moderate resolution significant differences from W0047+68 are revealed. Allers \& Liu (2013a) found that its moderate-resolution infrared spectrum lacked signs of low surface gravity or youth, and classified it as L6 FLD-G. This spectrum is compared to W0047+68 in Figure 1. With a reliable, precise distance, we can now revisit the kinematic arguments. Our measurement of the $U$-component of its space motion $\left(-34 \mathrm{~km} \mathrm{~s}^{-1}\right)$ is not consistent with any known young moving groups, but is not unusual among a sample of stars that are $\sim 0.5-2$ billion years old (such as dMe dwarfs or $M_{V}<4$ main-sequence stars; Reid et al. 2002, their Figure 15). Allende Prieto et al. (2004) measured [Fe/H] of all (118) stars more luminous than $M_{V}=6.5 \mathrm{mag}$ within $14.5 \mathrm{pc}$ from the Sun and found that $2 \%-5 \%$ have $[\mathrm{Fe} / \mathrm{H}]>+0.25$. Their most metal-rich star is HD 125072 which has $(U, V, W)=$ $(-37.9,-17.1,-34.8) \mathrm{km} \mathrm{s}^{-1}$, proving 2M2148+40's $U$ motion cannot be used to rule out high metallicity. Indeed, the $U$ velocity is consistent with the most common velocities of the very metal rich planet-host stars in the solar neighborhood, which are thought to be scattered from more inner regions of the Galactic disk (Ecuvillon et al. 2007). Santos et al. (2008) find that the nearby star-forming regions have near-solar metallicity in both $[\mathrm{Fe} / \mathrm{H}]$ and $[\mathrm{Si} / \mathrm{Fe}]$, and argue that high metallicity stars originate in the inner galaxy, and Wielen et al. (1996) show that orbital diffusion of metal-rich stars explains the presence of high metallicity stars locally. Wielen (1977) find that the typical change in the Galactic orbit radius will be $0.7-0.9 \mathrm{kpc}$ in $0.5-1.0 \mathrm{Gyr}$, the oldest ages consistent with the presence of lithium, but presumably 2M2148+40 would be an example of an unusually large change.

Given the observed luminosity, $T_{\text {eff }}=1507 \pm 11 \mathrm{~K}$ for $R=0.1 R_{\odot}$. Applying the DUSTY (Chabrier et al. 2000) evolutionary models to this luminosity and the observed presence of lithium, $2 \mathrm{M} 2148+40$ would have $M \approx 0.04-0.055 M_{\odot}$ and $\log g=5.0-5.2$ for ages 0.5 to 1.0 Gyr. Our luminosity for 2M2148+40 is similar to those of "normal" field L6 and L7 dwarfs (Vrba et al. 2004; Golimowski et al. 2004), so we necessarily derive similar effective temperatures. In summary, the new astrometry is consistent with the existing paradigm that $2 \mathrm{M} 2148+40$ has a normal surface gravity and effective temperature for a lithium L6-type dwarf, so that high metallicity (or else some other unknown factor) is needed to explain its unusually thick condensate clouds.

\section{CONCLUSIONS}

Our new measurements of W0047+68 confirm the view that it is an intermediate gravity ( $\log g \approx 4.5)$, young brown dwarf in the ABDMG. Relatively bright with a reliable age, metallicity, and luminosity, it is a key benchmark for characterizing the effects of clouds in substellar atmospheres. No theoretical model is fully satisfactory in matching both the optical and nearinfrared spectra and luminosity, but many features are explained and the need for increased mineral clouds is clear. The low effective temperature $(\sim 1300 \mathrm{~K})$ of $\mathrm{W} 0047+68$ reinforces the finding that the standard L/T transition sequence for field brown dwarfs $(\log g \approx 5)$ does not apply at intermediate gravities $(\log g=4.5)$, just as is already known for the low gravities of planetary mass objects $(\log g \approx 4.0)$, and the near-solar metallicity of the ABDMG demonstrates that gravity alone is responsible for the differing cloud properties. The luminosity and temperature of the older L dwarf $2 \mathrm{M} 2148+40$ is warmer than the L/T transition, but another factor, presumably metallicity, places it in the redder sequence of the color-magnitude diagram among the young objects.

We are grateful to the USNO CCD parallax team for allowing us to use the preliminary parallax measurements. We thank Eric Mamajek for discussions of local associations; Kimberly Aller for examining Pan-STARRS data; Denise Stephens, Satoko Sorohana, and Dagny Looper for providing electronic copies 
of their data; and France Allard and Adam Burrows for sharing their grids of synthetic spectra. This research has benefitted from the SpeX Prism Spectral Libraries, maintained by Adam Burgasser at http://www.browndwarfs.org/spexprism.

Some of the observations reported here were obtained at the MMT Observatory, a joint facility of the Smithsonian Institution and the University of Arizona. MMT telescope time was granted by NOAO, through the Telescope System Instrumentation Program (TSIP). TSIP is funded by NSF. Based on observations obtained at the Gemini Observatory, which is operated by the Association of Universities for Research in Astronomy (AURA) under a cooperative agreement with the NSF on behalf of the Gemini partnership: the National Science Foundation (United States), the Science and Technology Facilities Council (United Kingdom), the National Research Council (Canada), CONICYT (Chile), the Australian Research Council (Australia), CNPq (Brazil), and CONICET (Argentina). Some of the data presented herein were obtained at the W.M. Keck Observatory, which is operated as a scientific partnership among the California Institute of Technology, the University of California, and the National Aeronautics and Space Administration. The Observatory was made possible by the generous financial support of the W.M. Keck Foundation. The Pan-STARRS1 surveys have been made possible by the Institute for Astronomy, the University of Hawaii, the Pan-STARRS Project Office, the institutions of the Pan-STARRS1 Science Consortium (http://www.ps1sc.org), NSF, and NASA. This research has made use of NASA's Astrophysics Data System, the VizieR catalogue access tool, CDS, Strasbourg, France, and the NASA/IPAC Infrared Science Archive, which is operated by the Jet Propulsion Laboratory, California Institute of Technology, under contract with the National Aeronautics and Space Administration. IRAF is distributed by the National Optical Astronomy Observatory, which is operated by the Association of Universities for Research in Astronomy (AURA) under cooperative agreement with the National Science Foundation.

\section{REFERENCES}

Ackerman, A. S., \& Marley, M. S. 2001, ApJ, 556, 872

Allard, F., Homeier, D., \& Freytag, B. 2011, in ASP Conf. Ser. 448, 16th Cambridge Workshop on Cool Stars, Stellar Systems, and the Sun, ed. C. Johns-Krull, M. K. Browning, \& A. A. West (San Francisco, CA: ASP), 91

Allard, F., Homeier, D., \& Freytag, B. 2012, RSPTA, 370, 2765

Allard, F., Homeier, D., Freytag, B., Schaffenberger, W., \& Rajpurohit, A. S. 2013, MSAIS, 24, 128

Allende Prieto, C., Barklem, P. S., Lambert, D. L., \& Cunha, K. 2004, A\&A, 420,183

Allers, K. N., \& Liu, M. C. 2013a, ApJ, 772, 79

Allers, K. N., \& Liu, M. C. 2013b, MmSAI, 84, 1089

Allers, K. N., Liu, M. C., Dupuy, T. J., \& Cushing, M. C. 2010, ApJ, 715, 561

Antoja, T., Figueras, F., Fernández, D., \& Torra, J. 2008, A\&A, 490, 135

Apai, D., Radigan, J., Buenzli, E., et al. 2013, ApJ, 768, 121

Artigau, É., Bouchard, S., Doyon, R., \& Lafrenière, D. 2009, ApJ, 701, 1534

Baraffe, I., Chabrier, G., Allard, F., \& Hauschildt, P. H. 2002, A\&A, 382, 563

Barenfeld, S. A., Bubar, E. J., Mamajek, E. E., \& Young, P. A. 2013, ApJ, 766, 6

Barman, T. S., Macintosh, B., Konopacky, Q. M., \& Marois, C. 2011, ApJ, 733,65

Biazzo, K., D’Orazi, V., Desidera, S., et al. 2012, MNRAS, 427, 2905

Bihain, G., Rebolo, R., Zapatero Osorio, M. R., Béjar, V. J. S., \& Caballero, J. A. 2010, A\&A, 519, A93

Blake, C. H., Charbonneau, D., \& White, R. J. 2010, ApJ, 723, 684

Bovy, J., Hogg, D. W., \& Roweis, S. T. 2009, ApJ, 700, 1794

Bowler, B. P., Liu, M. C., Dupuy, T. J., \& Cushing, M. C. 2010, ApJ, 723, 850

Bowler, B. P., Liu, M. C., Shkolnik, E. L., \& Dupuy, T. J. 2013, ApJ, 774, 55

Bowler, B. P., Liu, M. C., Shkolnik, E. L., et al. 2012, ApJ, 753, 142
Burgasser, A. J., Geballe, T. R., Leggett, S. K., Kirkpatrick, J. D., \& Golimowski, D. A. 2006, ApJ, 637, 1067

Burrows, A., Heng, K., \& Nampaisarn, T. 2011, ApJ, 736, 47

Burrows, A., Marley, M., Hubbard, W. B., et al. 1997, ApJ, 491, 856

Burrows, A., Sudarsky, D., \& Hubeny, I. 2006, ApJ, 640, 1063

Chabrier, G., Baraffe, I., Allard, F., \& Hauschildt, P. 2000, ApJ, 542, 464

Chauvin, G., Lagrange, A., Dumas, C., et al. 2004, A\&A, 425, L29

Cruz, K. L., Kirkpatrick, J. D., \& Burgasser, A. J. 2009, AJ, 137, 3345

Currie, T., Burrows, A., Madhusudhan, N., et al. 2013, ApJ, 776, 15

Cushing, M. C., Marley, M. S., Saumon, D., et al. 2008, ApJ, 678, 1372

Cushing, M. C., Vacca, W. D., \& Rayner, J. T. 2004, PASP, 116, 362

Dahn, C. C., Harris, H. C., Vrba, F. J., et al. 2002, AJ, 124, 1170

Delorme, P., Gagné, J., Malo, L., et al. 2012, A\&A, 548, A26

Dupuy, T. J., \& Liu, M. C. 2012, ApJS, 201, 19

Ecuvillon, A., Israelian, G., Pont, F., Santos, N. C., \& Mayor, M. 2007, A\&A, 461,171

Eggen, O. J. 1992, AJ, 103, 1302

Elston, R., Raines, S. N., Hanna, K. T., et al. 2003, Proc. SPIE, 4841, 1611

Faherty, J. K., Burgasser, A. J., Walter, F. M., et al. 2012, ApJ, 752, 56

Faherty, J. K., Cruz, K. L., Rice, E. L., \& Riedel, A. 2013a, in Protostars and Planets VI, Poster, \#2G024

Faherty, J. K., Rice, E. L., Cruz, K. L., Mamajek, E. E., \& Núñez, A. 2013b, AJ, 145,2

Freytag, B., Allard, F., Ludwig, H.-G., Homeier, D., \& Steffen, M. 2010, A\&A, 513, A19

Gagné, J., Lafrenière, D., Doyon, R., Malo, L., \& Artigau, É. 2014, ApJ, 783,121

Gizis, J. E., Faherty, J. K., Liu, M. C., et al. 2012, AJ, 144, 94

Gizis, J. E., Jao, W., Subasavage, J. P., \& Henry, T. J. 2007, ApJL, 669, L45

Golimowski, D. A., Leggett, S. K., Marley, M. S., et al. 2004, AJ, 127, 3516

Helling, C., Ackerman, A., Allard, F., et al. 2008a, in IAU Symp. 249

Exoplanets: Detection, Formation and Dynamics, ed. Y.-S. Sun, S. Ferraz-

Mello, \& J.-L. Zhou (Cambridge: Cambridge Univ. Press), 173

Helling, C., Woitke, P., \& Thi, W.-F. 2008b, A\&A, 485, 547

Hook, I. M., Jørgensen, I., Allington-Smith, J. R., et al. 2004, PASP, 116, 425

Jeffries, R. D. 1995, MNRAS, 273, 559

Kaiser, N., Burgett, W., Chambers, K., et al. 2010, Proc. SPIE., 7733, 77330E

Kirkpatrick, J. D. 2005, ARA\&A, 43, 195

Kirkpatrick, J. D., Cruz, K. L., Barman, T. S., et al. 2008, ApJ, 689, 1295

Kirkpatrick, J. D., Gelino, C. R., Cushing, M. C., et al. 2012, ApJ, 753, 156

Kirkpatrick, J. D., Reid, I. N., Liebert, J., et al. 1999, ApJ, 519, 802

Kirkpatrick, J. D., Reid, I. N., Liebert, J., et al. 2000, AJ, 120, 447

Knapp, G. R., Leggett, S. K., Fan, X., et al. 2004, AJ, 127, 3553

Konopacky, Q. M., Barman, T. S., Macintosh, B. A., \& Marois, C. 2013, Sci, 339, 1398

Leggett, S. K., Saumon, D., Marley, M. S., et al. 2007, ApJ, 655, 1079

Liu, M. C., Dupuy, T. J., \& Allers, K. N. 2013a, AN, 334, 85

Liu, M. C., Magnier, E. A., Deacon, N. R., et al. 2013b, ApJL, 777, L20

Livingston, W., \& Wallace, L. 1991, An Atlas of the Solar Spectrum in the Infrared from 1850 to $9000 \mathrm{~cm}^{-1}$ (1.1 to 5.4 micrometer) (Tucson, AZ: National Solar Observatory)

Lodders, K. 1999, ApJ, 519, 793

Lodders, K., \& Fegley, B. 2002, Icar, 155, 393

Looper, D. L., Kirkpatrick, J. D., Cutri, R. M., et al. 2008, ApJ, 686, 528

López-Santiago, J., Montes, D., Crespo-Chacón, I., \& Fernández-Figueroa, M. J. 2006, ApJ, 643, 1160

Luhman, K. L., Stauffer, J. R., \& Mamajek, E. E. 2005, ApJL, 628, L69

Madhusudhan, N., Burrows, A., \& Currie, T. 2011, ApJ, 737, 34

Makarov, V. V. 2003, AJ, 126, 1996

Manjavacas, E., Bonnefoy, M., Schlieder, J. E., et al. 2014, A\&A, 564, A55

Marley, M. S., Saumon, D., Cushing, M., et al. 2012, ApJ, 754, 135

Marley, M. S., Seager, S., Saumon, D., et al. 2002, ApJ, 568, 335

Marocco, F., Day-Jones, A. C., Lucas, P. W., et al. 2014, MNRAS, 439, 372

Marois, C., Macintosh, B., Barman, T., et al. 2008, Sci, 322, 1348

Marois, C., Zuckerman, B., Konopacky, Q. M., Macintosh, B., \& Barman, T. 2010, Natur, 468, 1080

McLean, I. S., Becklin, E. E., Bendiksen, O., et al. 1998, Proc. SPIE, 3354, 566 McLean, I. S., Graham, J. R., Becklin, E. E., et al. 2000, Proc. SPIE, 4008, 1048

Montes, D., López-Santiago, J., Gálvez, M. C., et al. 2001, MNRAS, 328, 45 Oppenheimer, B. R., Baranec, C., Beichman, C., et al. 2013, ApJ, 768, 24

Ortega, V. G., Jilinski, E., de La Reza, R., \& Bazzanella, B. 2007, MNRAS, 377,441

Patience, J., King, R. R., de Rosa, R. J., \& Marois, C. 2010, A\&A, 517, A76

Patience, J., King, R. R., De Rosa, R. J., et al. 2012, A\&A, 540, A85

Pavlenko, Y., Zapatero Osorio, M. R., \& Rebolo, R. 2000, A\&A, 355, 245

Radigan, J., Jayawardhana, R., Lafrenière, D., et al. 2012, ApJ, 750, 105

Rayner, J. T., Toomey, D. W., Onaka, P. M., et al. 2003, PASP, 115, 362 
Reid, I. N., Gizis, J. E., \& Hawley, S. L. 2002, AJ, 124, 2721

Santos, N. C., Melo, C., James, D. J., et al. 2008, A\&A, 480, 889

Skemer, A. J., Hinz, P. M., Esposito, S., et al. 2012, ApJ, 753, 14

Soderblom, D. R., Laskar, T., Valenti, J. A., Stauffer, J. R., \& Rebull, L. M. 2009, AJ, 138, 1292

Sorahana, S., \& Yamamura, I. 2012, ApJ, 760, 151

Sorahana, S., Yamamura, I., \& Murakami, H. 2013, ApJ, 767, 77

Spiegel, D. S., Burrows, A., \& Milsom, J. A. 2011, ApJ, 727, 57

Stephens, D. C., Leggett, S. K., Cushing, M. C., et al. 2009, ApJ, 702, 154

Thompson, M. A., Kirkpatrick, J. D., Mace, G. N., et al. 2013, PASP, 125,809

Tonry, J. L., Stubbs, C. W., Lykke, K. R., et al. 2012, ApJ, 750, 99

Torres, C. A. O., Quast, G. R., Melo, C. H. F., \& Sterzik, M. F. 2008, Young Nearby Loose Associations (San Francisco, CA: Astronomical Society of the Pacific), 757
Tsuji, T. 2005, ApJ, 621, 1033

Vacca, W. D., Cushing, M. C., \& Rayner, J. T. 2003, PASP, 115, 389

Viana Almeida, P., Santos, N. C., Melo, C., et al. 2009, A\&A, 501, 965

Visscher, C., Lodders, K., \& Fegley, B., Jr. 2010, ApJ, 716, 1060

Vrba, F. J., Henden, A. A., Luginbuhl, C. B., et al. 2004, AJ, 127, 2948

Wahhaj, Z., Liu, M. C., Biller, B. A., et al. 2011, ApJ, 729, 139

Wielen, R. 1977, A\&A, 60, 263

Wielen, R., Fuchs, B., \& Dettbarn, C. 1996, A\&A, 314, 438

Witte, S., Helling, C., Barman, T., Heidrich, N., \& Hauschildt, P. H. 2011, A\&A, 529, A44

Witte, S., Helling, C., \& Hauschildt, P. H. 2009, A\&A, 506, 1367

Zuckerman, B., Song, I., \& Bessell, M. S. 2004, ApJL, 613, L65

Zuckerman, B., Song, I., Bessell, M. S., \& Webb, R. A. 2001, ApJL, 562, L87 